San Jose State University

SJSU ScholarWorks

Master's Theses

Master's Theses and Graduate Research

Summer 2019

\title{
Effect of Nicotine Exposure on Fatigue Mechanics of Murine Arteries
}

Elizabeth Ho

San Jose State University

Follow this and additional works at: https://scholarworks.sjsu.edu/etd_theses

\section{Recommended Citation}

Ho, Elizabeth, "Effect of Nicotine Exposure on Fatigue Mechanics of Murine Arteries" (2019). Master's Theses. 5034.

DOI: https://doi.org/10.31979/etd.qyz4-zb6f

https://scholarworks.sjsu.edu/etd_theses/5034

This Thesis is brought to you for free and open access by the Master's Theses and Graduate Research at SJSU ScholarWorks. It has been accepted for inclusion in Master's Theses by an authorized administrator of SJSU ScholarWorks. For more information, please contact scholarworks@sjsu.edu. 
EFFECT OF NICOTINE EXPOSURE ON FATIGUE MECHANICS

OF MURINE ARTERIES

\author{
A Thesis \\ Presented to \\ The Faculty of the Department of Mechanical Engineering \\ San José State University \\ In Partial Fulfillment \\ of the Requirements for the Degree \\ Master of Science
}

by

Elizabeth Ho

August 2019 
(C) 2019

Elizabeth Ho

ALL RIGHTS RESERVED 
The Designated Thesis Committee Approves the Thesis Titled

\title{
EFFECT OF NICOTINE EXPOSURE ON FATIGUE MECHANICS OF MURINE ARTERIES
}

by

Elizabeth Ho

APPROVED FOR THE DEPARTMENT OF MECHANICAL ENGINEERING

\author{
SAN JOSÉ STATE UNIVERSITY
}

August 2019

Sang-Joon Lee, Ph.D.

Anand Ramasubramanian, Ph.D.

Joscha Udo Nikolaus Mulorz, Ph.D.
Department of Mechanical Engineering Department of Chemical and Materials Engineering

Postdoctoral Research Fellow, Cardiovascular Medicine, Stanford University 


\section{ABSTRACT \\ EFFECT OF NICOTINE EXPOSURE ON FATIGUE MECHANICS \\ OF MURINE ARTERIES}

by Elizabeth Ho

Nicotine is an addictive substance found in electronic cigarettes (e-cigarettes) and cigarettes, and the use of such products has growing concern because of the prevalent use by young adults. Nicotine exposure degrades arterial tissue and results in increased arterial stiffness, which is linked to cardiovascular events, such as stroke and myocardial infarction. The hypothesis of this research is that fatigue loading reveals changes to the mechanical behavior of nicotine-treated arteries that is more informatively discriminating than stiffness alone. Ten murine arteries, five untreated and five nicotine-treated, were subjected to cyclic fatigue loading in an open-circumferential configuration by a custom tensometer with a motorized actuator and inline load cell. The specimens were subjected to an alternating strain of $\pm 50 \%$, superimposed on a mean strain of $150 \%$. A power law was fit to the experimental data to extract parameters indicative of peak stress, loss of tension, degradation slope, and oscillation band. Compared to untreated specimens, nicotine-treated specimens exhibited a 108\% higher peak stress, $118 \%$ greater loss of tension, and 107\% larger width of the oscillation band. The tension loss and oscillation band width provided additional discriminating evidence of different mechanical behavior, supporting the hypothesis that fatigue testing can reveal distinctions in mechanical behavior that are not evident in static testing alone. 


\section{ACKNOWLEDGMENTS}

I would like to thank my committee members Dr. Sang-Joon Lee, Dr. Anand Ramasubramanian, and Dr. Joscha Udo Nikolaus Mulorz on their overall support and technical guidance of this project. Specifically, I would like to thank my chair Dr. Sang-Joon Lee for encouraging me to pursue my thesis early on in my graduate career and constantly challenging me to work to the best of my abilities. I would like to thank Dr. Philip Tsao and his research group for insights on the scientific merit of this type of experimental investigation. I would like to thank Wilson Eng on his efforts in developing the custom apparatus used in this project. I would like to thank Sue-Mae Saw and Chaitanya Puligundla for their assistance on my experimentation. I would like to thank my friends and family for all their support during the journey of this publication. 


\section{TABLE OF CONTENTS}

List of Tables.............................................................................

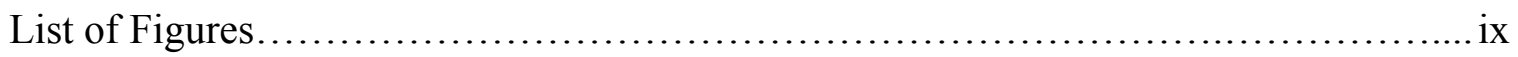

List of Symbols and Abbreviations................................................ xi

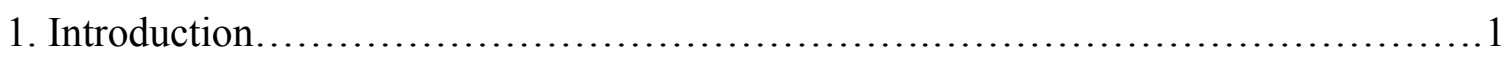

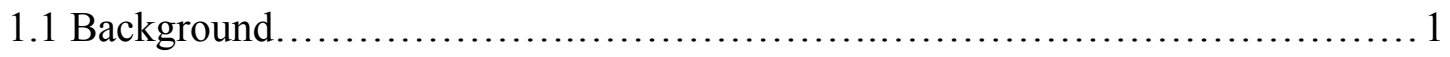

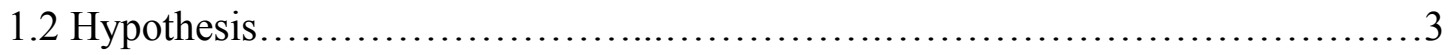

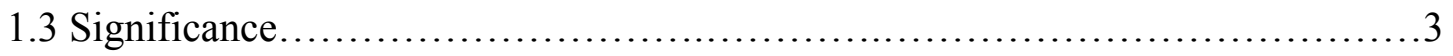

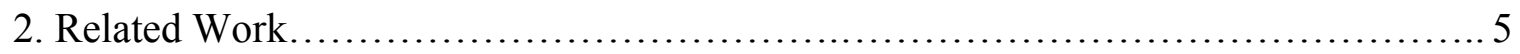

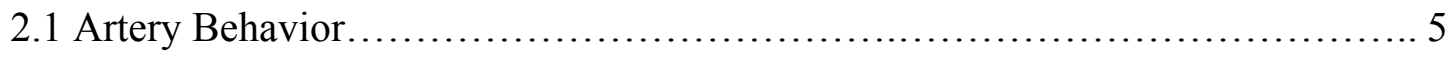

2.1.1 Preconditioning Behavior............................................... 5

2.1.2 Stress to Stretch Behavior.............................................. 6

2.1.3 Fiber Orientation...................................................... 8

2.1.4 Constitutive Modeling.................................................. 9

2.1.5 Fatigue of Tissue...................................................... 10

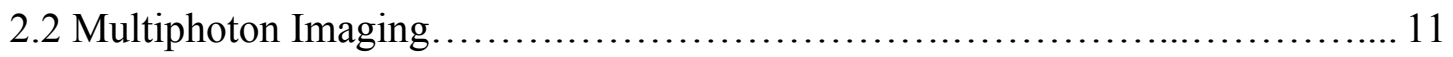

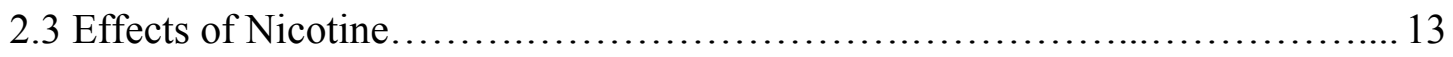

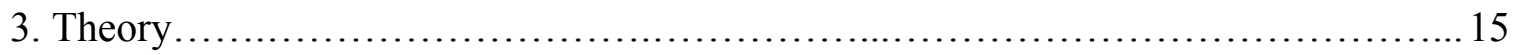

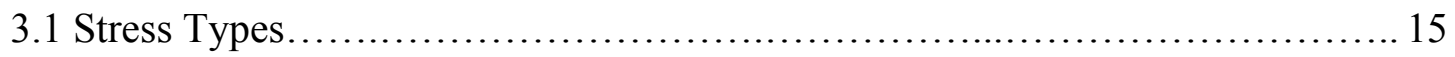

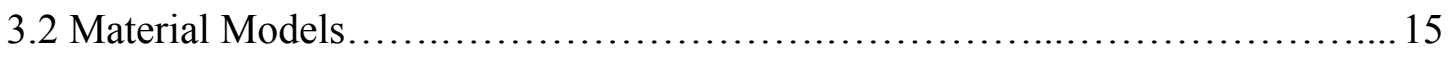

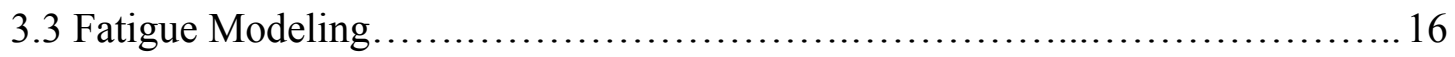

3.4 Experimental Uncertainty ................................................ 17

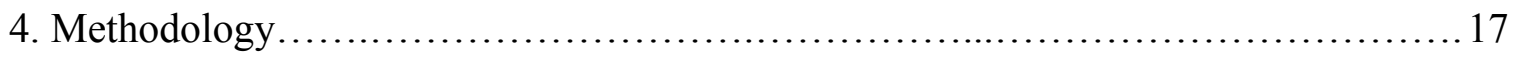

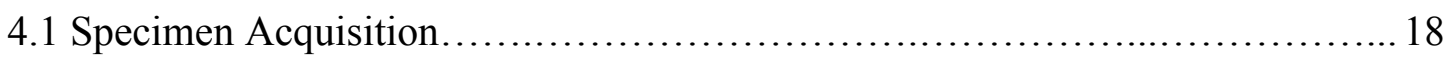

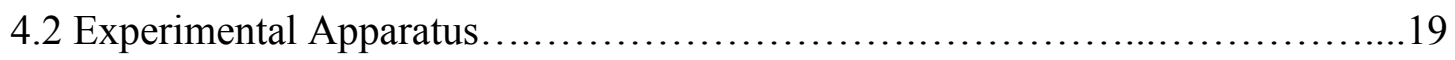

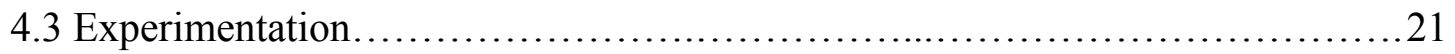

4.3.1 Fatigue Testing Protocol............................................... 21

4.3.2 Multiphoton Microscopy Protocol...........................................22

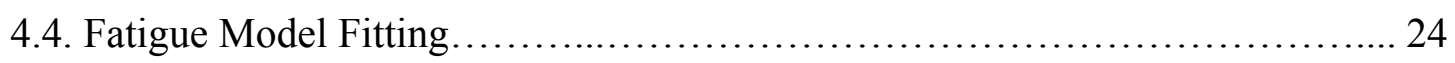

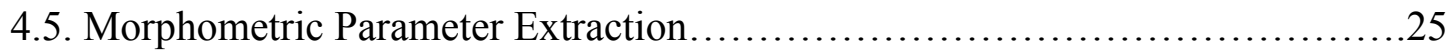




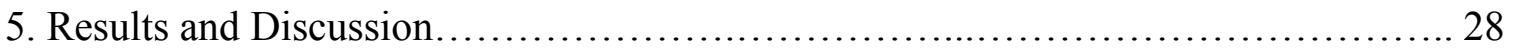

5.1 Untreated and Nicotine-Treated Fatigue Protocol Data.........................28

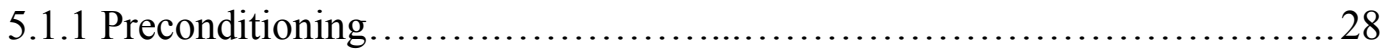

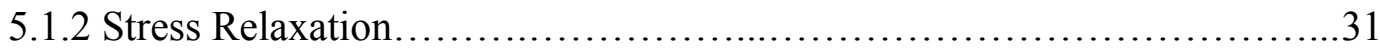

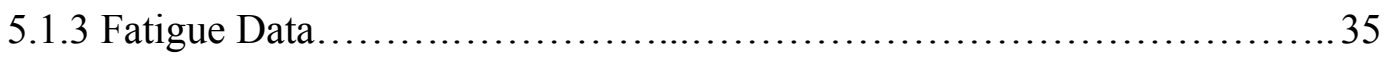

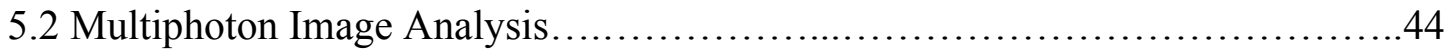

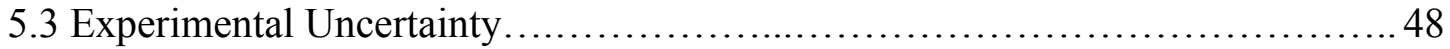

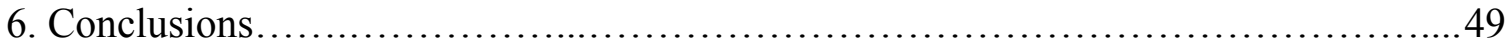

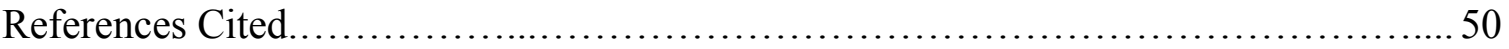

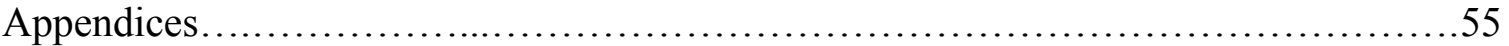

Appendix A: Aluminum Clamps Fabrication Drawings........................... 55

Appendix B: Raw Data of Fit Parameters...................................... 56 


\section{LIST OF TABLES}

Table 1. Power Law Constants For Untreated Specimens............... 34

Table 2. Power Law Constants For Nicotine-Treated Specimens...... 35

Table 3. $\quad$ Parameters $A, B, C$, and $D$ Extracted For Untreated 38

Specimens..........................................

Table 4. $\quad$ Parameters $A, B, C$, and $D$ Extracted For Nicotine-Treated 38

Specimens............................................

Table 5. Average Values Of Fatigue Parameters For Untreated And 43

Nicotine-Treated Specimens..............................

Table 6. Sources Of Experimental Uncertainty..................... 48 


\section{LIST OF FIGURES}

Figure 1. $\quad$ Labeled schematic diagram of specimen set-up............ 20

Figure 2. Dimensional drawing of aluminum clamps used to secure $\quad 21$

Figure 3. Preconditioning plots for untreated murine arteries.......... 29

Figure 4. Preconditioning plots for nicotine-treated murine arteries... $\quad 30$

Figure 5. Stress relaxation plots for untreated thoracic arteries........ 31

Figure 6. Stress relaxation plots for nicotine-treated thoracic arteries.. $\quad 32$

Figure 7. Stress relaxation plots with fitted lines for untreated 33

thoracic arteries........................................

Figure 8. Stress relaxation plots with fitted lines for nicotine-treated 34

thoracic arteries.......................................

Figure 9. Fatigue data for untreated specimens................... 36

Figure 10. Fatigue data for nicotine-treated specimens............... 37

Figure 11. Scatter plots of parameters extracted from fatigue model for $\quad 39$

untreated and nicotine-treated specimens..................

Figure 12. Fatigue data with fitted lines for untreated thoracic arteries.. $\quad 40$

Figure 13. Fatigue data with fitted lines for nicotine-treated thoracic 41

Figure 14. Simulated model of fatigue output.................... 42

Figure 15. $\quad$ Effects on simulated model of fatigue after an increase in $\quad 42$ value of individual parameters $A(\mathrm{a}), B(\mathrm{~b}), C(\mathrm{c})$, and $D(\mathrm{~d})$

Figure 16. Multiphoton elastin images for unstretched untreated 44

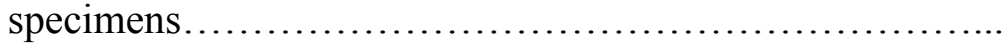

Figure 17. Multiphoton elastin images for unstretched nicotine-treated 45 specimens......................................... 
Figure 18. Multiphoton elastin image (left), binarized image (middle), and skeletonized image (right) for Unstretched Untreated

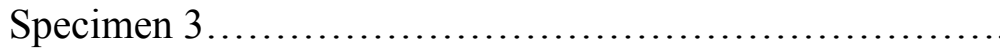

Figure 19. Multiphoton elastin image (left), binarized image (middle), and skeletonized image (right) for Unstretched Nicotine-Treated Specimen $5 \ldots \ldots \ldots \ldots \ldots \ldots \ldots \ldots \ldots \ldots \ldots$

Figure 20. Scatter plots of morphometric parameters extracted from multiphoton images for unstretched untreated and unstretched nicotine-treated specimens..................... 


\section{LIST OF SYMBOLS AND ABBREVIATIONS}

\begin{tabular}{|c|c|c|}
\hline Symbol & Units & Description \\
\hline$a$ & $\mathrm{~Pa}$ & First fatigue material parameter (S-N diagram) \\
\hline$a_{1}$ & - & First power law parameter \\
\hline$A$ & $\mathrm{~Pa}$ & First fatigue material parameter (model proposed in study) \\
\hline$A_{0}$ & $\mathrm{~mm}^{2}$ & Area of original cross-section \\
\hline$A_{1}$ & $\mathrm{~mm}^{2}$ & Area of deformed cross-section \\
\hline$b$ & - & First fatigue material parameter by Hwang and Han et al. [28] \\
\hline$b_{1}$ & - & Second power law parameter \\
\hline$B$ & $\mathrm{~Pa}$ & Second fatigue material parameter (model proposed in study) \\
\hline$c$ & - & Second fatigue material parameter by Hwang and Han et al. [28] \\
\hline$C$ & - & Third fatigue material parameter (model proposed in study) \\
\hline$C_{10}$ & $\mathrm{~Pa}$ & First Mooney-Rivlin parameter \\
\hline$C_{01}$ & $\mathrm{~Pa}$ & Second Mooney-Rivlin parameter \\
\hline$d$ & - & Second fatigue material parameter (S-N diagram) \\
\hline$D$ & - & Fourth fatigue material parameter (model proposed in study) \\
\hline$E_{1}$ & $\mathrm{~Pa}$ & Young's modulus of first material \\
\hline$E_{2}$ & $\mathrm{~Pa}$ & Young's modulus of second material \\
\hline$f$ & $\mathrm{~N}$ & Resultant force on cross-section \\
\hline$F$ & - & Applied force \\
\hline$g(t)$ & $\mathrm{Pa}$ & Step temporal response \\
\hline$h$ & $\mathrm{~mm}$ & Thickness of specimen \\
\hline$k_{e q}$ & $\mathrm{~N} / \mathrm{m}$ & Equivalent stiffness of system \\
\hline$k_{1}$ & $\mathrm{~N} / \mathrm{m}$ & Stiffness of load cell \\
\hline$k_{2}$ & $\mathrm{~N} / \mathrm{m}$ & Stiffness of specimen \\
\hline$l$ & $\mathrm{~m}$ & Length of specimen \\
\hline$N$ & - & Number of cycles \\
\hline$r$ & - & Applied stress \\
\hline$S$ & $\mathrm{~Pa}$ & Stress (model proposed in study) \\
\hline$S_{f}$ & $\mathrm{~Pa}$ & Fatigue strength (S-N diagram) \\
\hline$t$ & s & Time \\
\hline$u(t)$ & - & Unit step function \\
\hline$W$ & $\mathrm{~Pa}$ & Strain-energy density \\
\hline$\lambda$ & - & Ratio of deformed specimen length to original specimen length \\
\hline$\sigma$ & $\mathrm{Pa}$ & Cauchy stress, or true stress \\
\hline$\sigma_{2 P K}$ & $\mathrm{~Pa}$ & Second Piola-Kirchhoff stress \\
\hline
\end{tabular}




$\begin{array}{lll}\sigma_{a} & \mathrm{~Pa} & \text { Alternating stress } \\ \sigma_{m} & \mathrm{~Pa} & \text { Mean stress } \\ \sigma_{\text {nom }} & \mathrm{Pa} & \text { Nominal stress, or engineering stress } \\ \tau & \mathrm{s} & \text { Time constant }\end{array}$

$\begin{array}{ll}\text { Abbreviation } & \text { Full Spelling } \\ \text { 2PEF } & \text { Two-photon excited fluorescence } \\ \text { AAA } & \text { Abdominal aortic aneurysm } \\ \text { DAQ } & \text { Data acquisition system } \\ \text { ECM } & \text { Extracellular matrix } \\ \text { MMP } & \text { Matrix metalloproteinases } \\ \text { PAVIR } & \text { Palo Alto Veterans Institute for Research } \\ \text { RGB } & \text { Red-green-blue true color } \\ \text { RWS } & \text { Relative wall strain } \\ \text { SD } & \text { Standard devation } \\ \text { SE } & \text { Structural element } \\ \text { SHG } & \text { Second harmonic generation } \\ \text { VAPAHCS } & \text { VA Palo Alto Health Care System } \\ \text { WSS } & \text { Wall shear stress }\end{array}$




\section{INTRODUCTION}

\subsection{Background}

The study of arterial mechanics has been of interest in attempts to predict the onset of hypertension, aneurysms, or other cardiovascular diseases. In the human body, the aorta is the main vessel that continuously receives blood from the heart and supplies blood to the entire systemic cardiovascular system. This means that the aorta must exhibit an elastic behavior that will expand to comply to the stress exerted by the surge of blood flow from the left ventricle and then recoil to push the blood through the body, otherwise referenced as the Windkessel function [1]. Throughout a human lifetime, arteries experience cyclic loading of blood as the heart pumps, exerting cyclic circumferential stress on the vessel walls. As additional stresses and external factors are exerted on the artery, the stiffness of the arterial wall increases. An ingredient that is linked to aortic stiffening is nicotine, an addictive substance found in products such as cigarettes or e-cigarettes. Nicotine exposure has been shown to increase the degradation of elastin, reducing the elasticity of and increasing the stiffness of arteries.

Increased stiffness and decreased distensibility of the artery are commonly found to influence the onset of cardiovascular events, such as stroke, hypertension, or myocardial infarction [2]. Arterial wall remodeling occurs under changes in loading, which impacts the stiffness and compliance of the artery. Arteries with increased stiffness are associated with a lack of damping properties and correlate with the formation of abdominal aortic aneurysms [1]. Hemodynamic forces, such as wall shear stress (WSS) and relative wall 
strain (RWS), play a factor in the formation of aneurysms. Enlarged blood vessel diameter is commonly known to detect the risk of aneurysm rupture. However, another factor that increases the likelihood of rupture is the increasing ratio of wall stress to wall strength of the aneurysm, or when the wall stress exceeds wall strength [3]. Thus, the understanding of both the mechanical properties and the microstructure of the arterial wall are helpful tools to predict the consequences of factors that are associated with the elevated risk of cardiovascular diseases.

The arterial wall is made up of three layers, intima, media, and adventitia. The intima does not contribute to the mechanical behavior of the artery, so the media and adventitia layers are the most relevant layers for tensile testing, as they contain the load-bearing components elastin and collagen [4], [5]. Arteries are considered anisotropic, so the loads applied at orthogonal directions produce different stress results from each other. This behavior is attributed to arteries containing both collagen and elastin fibers oriented in different directions in the media and the adventitia layers. Under mechanical deformation, the collagen and elastin networks reorient themselves in different directions based on the direction of pull.

The internal elastin and collagen fiber network of the arterial wall can be imaged using a multiphoton microscope. Multiphoton microscopy uses nonlinear interactions to generate high contrast images. Images of collagen and elastin are captured by the detection of second harmonic generation (SHG) and two-photon excited fluorescence (2PEF) signals, respectively. SHG signals are detected due to collagen, whereas $2 \mathrm{PEF}$ 
signals are detected due to elastin [6]. SHG images, which are green color-coded, and 2PEF images, which are red color-coded, can be captured with excitation wavelengths and band-pass emission filters. One advantage of using multiphoton microscopy is that biological tissues can be imaged without fixing the specimens and without the use of contrast agents [6].

\subsection{Hypothesis}

The hypothesis of this investigation is that fatigue loading reveals changes in the mechanical behavior of nicotine-treated arteries that would not be detectable under static loading.

\subsection{Significance}

E-cigarettes have become a popular outlet for adolescents. Studies show that the rate of e-cigarette use increased in high school students from $1.5 \%$, or 220,000 students, in 2011 to $20.8 \%$, or 3.1 million students, in 2018 [7]. Adolescent exposure to e-cigarettes is linked to cigarette use as an adult. The main addictive substance in e-cigarettes and cigarettes is nicotine, which has been shown to increase levels of aortic stiffness with exposure. The exposure of arteries to the nicotine substance alters the integrity of the arterial wall by promoting excess release of proteinases, degrading elastin networks.

The significance of the research is to model the profile of arteries and compare fatigue parameters extracted from the model for untreated and nicotine-treated specimens, and to compare morphometric parameters extracted from elastin based multiphoton microscopy images for untreated and nicotine-treated specimens. 
Additional significance of this research is captured in the methodology, in which cyclic fatigue testing of arteries is performed in an open-circumferential configuration. While test methods that keep the vessel intact are commonly used, such as pressure myography, leakage may occur through small side branches and leakage may also be exacerbated with the testing of specimens at supraphysiological conditions [8]. 


\section{RELATED WORK}

\subsection{Artery Behavior}

\subsubsection{Preconditioning Behavior}

Fung et al. [9] developed a pseudo strain energy function that approximates the behavior of the artery when subjected to internal pressure and axial stretching. Pressure dilation testing was performed on rabbit arteries to develop the model. It was found that arteries exhibit hysteresis under cyclic loading, indicating that arteries are inelastic materials. Artery specimens can be modeled by assuming their composition is made up of two materials, where each material represents the loading and unloading curve in a hysteresis plot. It was found that during cyclic loading, the stress-strain relationship of an artery is more defined after preconditioning cycles have been implemented [9]. Holzapfel et al. [4] subjected circumferential strips of media of the human carotid aorta to uniaxial cyclic loading and unloading, and observed stress softening behaviors. Arteries are claimed to be preconditioned when the amount of stress softening has been limited and when they display a nearly repeatable behavior during loading and unloading. Lally et al. [10] found no change in the behavior of porcine arteries after five preconditioning cycles under uniaxial and equibiaxial tension testing. Preconditioning cycles are important to perform on specimens prior to subsequent testing in order to remove any deformation history [11]. De Gelidi et al. [12] studied the role of preconditioning on the characterization of hyperelastic materials and concluded that stress-strain relationships displayed statistical significance when preconditioning was performed versus when 
preconditioning was neglected. Rates of pull for preconditioning vary among studies, but tissue subjected to repeated cycling becomes insensitive to the strain rate [13]. Zhang et al. [14] also concluded that linear viscoelastic biomaterials and other typical biological soft tissues can be characterized to show rate-insensitive hysteretic behavior.

\subsubsection{Stress to Stretch Behavior}

The mechanics of arteries allow greater stretch in the circumferential direction versus the axial direction. Ramachandra et al. [15] performed biaxial testing on pulmonary murine arteries with a series of tests. These tests included six cycles of preconditioning, two cycles of pressure-diameter tests at various axial stretches, and two cycles of force-stretch tests at various pressure levels. It was observed that the maximum stretch ratio in the axial direction was limited to 1.6 , while the stretch ratio in the circumferential direction was limited to 2.0. Labrosse et al. [16] performed pressurization tests from 0 $\mathrm{kPa}$ to $21 \mathrm{kPa}$ on human aortas, and found that the aortas recorded a mean circumferential stretch ratio of 1.25 and a mean axial stretch ratio of 1.13 at a pressure of $13.3 \mathrm{kPa}$.

In the artery, elastin contributes to elastic properties, while collagen contributes to the stiffness properties. Weisbecker et al. [8] performed uniaxial extension tests on circumferentially and axially aligned collagenase-treated and elastase-treated media specimens of the human aorta. Collagenase breaks down collagen fibers, while elastase breaks down elastin fibers of the artery. Collagenase-treated and elastase-treated specimens were subjected to stretch values at physiological and supraphysiological ranges. Collagenase-treated specimens did not exhibit continuous or discontinuous 
softening, while elastase-treated specimens exhibited both continuous and discontinuous softening. Dobrin et al. [17] studied the radial elastic properties in arteries treated with elastase and collagenase. Specimens treated with collagenase did not alter the compressive stress of the arterial wall, while specimens with elastase resulted in a decrease in radial compressive stress, indicating that elastin does contribute to the resistance properties of the artery. Distensibility, describes the elastic properties of arteries and is defined as "the relative change in volume per unit of pressure $([\Delta \mathrm{V} / \mathrm{V}] / \Delta \mathrm{P}) "[18]$. Decreased distensibility is correlated with influence the onset of cardiovascular events [2].

Exhibiting an anisotropic mechanical behavior, biaxial testing of arteries helps to distinguish the differences of mechanical properties in the axial and circumferential directions. A stress-to-stretch ratio curve of an artery exhibits three distinct regions. The first region shows that the artery exhibits high extension with low stress. With collagen exhibiting greater stiffness than elastin, elastin will take up loads at lower pressures due to having greater deformability than collagen [19]. The second region is a linear region, indicating recruitment and straightening of the collagen fibers, adding stiffness to the tissue. The third region indicates the onset of plasticity, where all collagen fibers have been recruited and straightened [10]. In the extracellular matrix (ECM), load bearing components are made up of medial elastin, medial collagen, and adventitia collagen. While adventitia collagen does not get recruited until after $20 \%$ strain, medial collagen is 
continuously being recruited. Medial elastin straightens at lower strains, but loading of elastin plateaus at $20 \%$ strain as adventitia collagen begins to take on the load [5].

\subsubsection{Fiber Orientation}

The anisotropic behavior of arteries is found to be a result of different fiber orientations across layers of the arterial wall. During mechanical loading, collagen and elastin within the layers of the arterial wall reorient in different directions.

Yu et al. [20] performed equi-biaxial and non-equi-biaxial testing on thoracic porcine arteries and analyzed images of the arterial wall, specifically the layers of the media, under multiphoton microscopy. It was observed that elastin fibers are uniformly aligned in the inner media, circumferentially aligned in the middle media, and axially aligned in the outer media. Krasny et al. [21] performed uniaxial loading on rabbit carotid arteries along the circumferential axis, axial axis, and along an axis $45^{\circ}$ with respect to the axial axis. Under uniaxial loads, the collagen and elastin networks rearranged themselves in their own way differently across arterial wall layers. Media collagen, media elastin, and adventitia elastin did not fully align towards the direction of pull, but adventitia collagen fully oriented itself to align to the loading directions. Media elastin underwent minimal changes under the various loading conditions, whereas the adventitia elastin reoriented under axial load, but remained a fibrous network under circumferential load [21]. Another study performed by Krasny et al. [11] showed that under two different loading scenarios, collagen fibers reoriented towards the strain direction while subjected to 
tension in the axial direction with constant pressure, and the orientation of collagen fibers remained unchanged while subjected to pressure inflation with constant axial stretch.

\subsubsection{Constitutive Modeling}

Many constitutive models have been proposed for the study of biological tissues and each model should include constituents that make up the composition of the tissue. These models should represent the response of biological tissue to mechanical loads [22]. Fung et al. [9] proposed a pseudo strain energy function that estimated close approximation to the internal pressure and axial stretching of rabbit arteries. The two dimensional exponential model does not incorporate radial stresses into the pseudo strain energy equation. Holzapfel et al. [4] developed a constitutive model which represented the artery as a two-layer thick-walled tube because the media-intima and adventitia layers contain different residual strains and stresses, which may have an effect on stress distributions exerted on the arterial wall. Medial collagen, medial elastin, and adventitia collagen have all been shown to play a load-bearing role in the ECM [5]. Wang et al. [23] proposed a constitutive model that incorporates the three ECM constituents. Under mechanical loading, medial collagen and medial elastin are immediately recruited, while adventitia collagen recruitment is delayed. Because of this, Wang et al. [23] added a recruitment function, proposed by Lanir et al. [24], to the model to represent the delayed adventitia collagen.

The response of hyperelastic materials can be described by different models, such as Mooney-Rivlin or neo-Hookean. Watton et al. [25] studied two different models that 
have been proposed to model elastin. These models include the neo-Hookean, and another proposed by Zulliger et al. [26], which is similar to the neo-Hookean but includes a larger exponent value. Both models were fitted to the data generated by uniaxial extension of an elastinous sheet, as well as the inflation and extension of a cylindrical elastinous tube. The model by Zulliger et al. [26] did not fit uniaxial extension data and exhibited an increasing modulus. Thus, Watton et al. [25] concluded that, even with limitations to the neo-Hookean model, the model accurately describes the mechanical response of elastin.

\subsubsection{Fatigue of Tissue}

Arteries are typically tested under biaxial tension due to the anisotropic behavior of the arteries. Fatigue testing of tissues is typically concerned with number of cycles to failure, which is plotted on a S-N graph and fitted to a log-linear curve, where the material parameters of the specimens are found from the slope [27]. Tensile fatigue testing of biological tissue has mostly been performed to understand number of cycles to failure, rather than the degradative process that precedes rupture. Hwang and Han et al. [28] proposed a new mathematical model for fatigue modulus degradation of composites, which modeled their experimental data much closer than the log-linear or power law equations.

Washington et al. [29] studied the effects of oxidation and glycation on porcine arteries when subjected to cyclic loading of $100 \mathrm{k}$ cycles in increments of $25 \mathrm{k}$ cycles. Oxidation and glycation treatments are known to correlate with arterial stiffening. The 
data were fitted against the fatigue modulus model presented by Hwang and Han et al. [28], the power law, and a linear model. The fatigue model was used to quantify the damage to the elastin network. For both treatments, the fatigue modulus and power law were not significant across number of cycles, but the linear model was found to be significant. Limitations in this experiment were that the models may have behaved differently depending on how elastin was isolated in the arteries and a longer cyclic duration may have been required to see significant differences.

\subsection{Multiphoton Imaging}

Multiphoton imaging is used to image the microstructure of the arterial walls by providing 3-D images (z-stack) and distinct color images without the need to fix or stain the artery. SHG and 2PEF microscopy can be utilized to visualize collagen and elastin fibers within the artery by exciting the signals to be detected. SHG signals are detected due to the presence of collagen, while TPF signals are detected due to the presence of elastin resulting in a green and red image, representing collagen and elastin, respectively [30]. Many studies have indicated that the application of multiphoton microscopy is highly promising to understand the microstructure of the arterial wall.

Stein et al. [31] developed an algorithm for the extraction of the fiber network from 3-dimensional (3-D) collagen confocal fluorescence images, which has shown the ability to generate the network geometry of known architecture. Parameters that can be extracted from the fiber network include fiber length, cross-link spacing, and persistence length. Bredfeldt et al. [32] took the algorithm developed by Stein et al. [31] and evaluated the 
collagen fiber extraction on SHG images of breast cancer tissue with various preprocessing filters. Bredfeldt et al. [32] determined that with a denoising filter based on the 2-dimensional (2-D) curvelet transform prior to fiber network extraction, the algorithm was able to extract more accurate fiber segmentations.

Analysis of collagen or elastin images is performed to understand the behavior of fibers within the arterial wall. Koch et al. [33] created a custom MATLAB (The MathWorks, Natick, MA, USA) script to quantify the microarchitecture of collagen and elastin in the human aorta. Beginning with a representative input image of collagen or elastin, each image underwent a series of MATLAB functions. The image was smoothed through median filtering (medfilt2), contrast-enhanced (adapthisteq), and then saturated at $1 \%$ of the extremes (imadjust). Continuing with morphological functions, such as dilating (imdilate) or eroding (imerode), prepared the image to undergo a thinning function (bwmorph( 'thin')) to generate a 1-pixel skeleton of the fibers. The software was able to analyze the skeleton of fibers and generate parameters, such as intersections, diameter distribution, or segment length [33].

Another parameter that can be extracted from the artery is the waviness parameter, which is defined as the ratio of the length of the straight line connecting the ends of the fiber bundle, $L_{\rho}$, to the total length of fiber bundle, $L_{f}$ The smaller the waviness value, the greater the waviness of the fiber [30], [34]. Rezakhaniha et al. [34] used a plug-in of the imaging software, ImageJ, called NeuronJ to manually trace the collagen fibers from end to end, and measure the length of the fiber bundle used to calculate waviness, which was 
found to not correlate with the orientation of the fibers. Boulesteix et al. [30] studied the successful capability of combining multiphoton microscopy with ex vivo mouse artery rings by imaging collagen and elastin using an upright microscope and determining the waviness of elastin through these images. Fiber tortuosity can be modeled as a sinusoidal curve and be defined as the ratio of the length of the fiber to the length of the straight line connecting the ends of the bundle, with an angle of $\theta$ that describes the amplitude of the curve [35].

Parameter extraction from microscopy images is performed in hopes to describe the mechanics of the tissue. Wan et al. [22] extracted fiber distribution data from multiphoton images across the adventitia. Two cases for material parameter extraction were performed. For the first case, structural information was determined by a nonlinear regression, while for the second case, fiber data were determined from multiphoton images. The inclusion of measured structural data improved model predictions of the tissue at a minor expense of the goodness-of-fit. Having known material parameters, the measurement of the fiber data may be sufficient for describing the mechanical response of the artery.

\subsection{Effects of Nicotine}

Cigarette smoking has been known to cause cardiovascular diseases, which indicates that nicotine may play a large factor in the development of these diseases. The effects of nicotine on arterial stiffening are studied in order to better understand the consequences of nicotine-delivery products, such as cigarettes and e-cigarettes, on the body. An 
increased level of nicotine has been linked to aortic stiffening and increased elastase activity [36]. Kool et al. [37] studied the short and long-term effects of smoking on the arterial wall of habitual smokers and found that smoking causes short-term arterial stiffening, but no significant long-term effects of smoking on the vessel wall. The long-term effect concluded from the study was higher heart rate.

Matrix metalloproteinases (MMPs) are connective tissue proteinases that can be found in the extracellular matrix. An increased amount of MMPs has been shown to be associated with the degradation of the extracellular matrix, specifically elastin in the aneurysm wall, and the onset of AAA formation [38]. Jacob-Ferreira et al. [39] studied the effects of nicotine on smooth muscle cells from rat aortas and concluded that nicotine exposure caused the release of MMPs by the smooth muscle cells and increased the valcular MMP activity. Wagenhäuser et al. [40] studied the effects of nicotine on arterial stiffness on abdominal and thoracic murine arteries, after subjected to nicotine exposure of 40 days. Stiffness increased in the abdominal segment and thoracic segment at 10 days and 40 days, respectively. Mice that were exposed to nicotine showed elevated levels of aortic stiffness as compared to the control group of mice infused with phosphate buffer solution (PBS). Nicotine effects were found to be greater in the abdominal segment because less elastin is found in the abdominal segment, as compared to the thoracic segment. With the increase of MMP due to nicotine, the degradation of elastin occurs, reducing the elastic properties and leading to the stiffening of the artery. 


\section{THEORY}

\subsection{Stress Types}

For tensile testing of soft biological tissues, the stress calculation must consider the tissue elasticity in reference to the area. Nominal stress, or engineering stress, is defined by Equation (1), where $f$ is the resultant force on the cross-section and $A_{0}$ is the area of the original cross-section.

$$
\sigma_{n o m}=\frac{f}{A_{0}}
$$

Cauchy stress, or true stress, is defined by Equation (2), where $f$ is the resultant force on the cross-section and $A_{1}$ is the area of the deformed cross-section.

$$
\sigma=\frac{f}{A_{1}}
$$

The second Piola-Kirchhoff stress is defined by Equation (3), where $\sigma_{\text {nom }}$ is the nominal stress and $\lambda$ is the ratio of deformed specimen length to original specimen length [41].

$$
\sigma_{2 P K}=\frac{\sigma_{n o m}}{\lambda}
$$

\subsection{Material Models}

Strain-energy functions have been proposed to model the properties of hyperelastic materials. The isotropic elastic properties of hyperelastic materials are modeled by Equation (4), where $W$ is strain-energy density, $I_{1}, I_{2}$, and $I_{3}$ are the invariants of two Cauchy-Green deformation tensors. 


$$
W=f\left(I_{1}, I_{2}, I_{3}\right)
$$

Invariants $I_{1}, I_{2}$, and $I_{3}$ are defined in Equations (5), (6), and (7), where $\lambda_{1}, \lambda_{2}$, and $\lambda_{3}$ are extension ratios.

$$
\begin{gathered}
I_{1}=\lambda_{1}^{2}+\lambda_{2}^{2}+\lambda_{3}^{2} \\
I_{2}=\lambda_{1}^{2} \lambda_{2}^{2}+\lambda_{2}^{2} \lambda_{3}^{2}+\lambda_{3}^{2} \lambda_{1}^{2} \\
I_{3}=\lambda_{1}^{2} \lambda_{2}^{2} \lambda_{3}^{2}
\end{gathered}
$$

The Mooney-Rivlin model is shown in Equation (8).

$$
W=C_{10}\left(I_{1}-3\right)+C_{01}\left(I_{2}-3\right)
$$

Reduction of the Mooney-Rivlin model results in the neo-Hookean model, shown in Equation (9). While both Mooney-Rivlin and neo-Hookean are commonly used for hyperelastic materials, Gundiah et al. [42] identified the neo-Hookean model as more appropriate for arterial elastin.

$$
W=C_{10}\left(I_{1}-3\right)
$$

\subsection{Fatigue Modeling}

Fatigue failure in metals occurs when the maximum stress exerted, which is typically lower than ultimate strength, is repeated many times. The fatigue strength of a material is typically presented on an S-N diagram with $S_{f}$ as fatigue strength in Pa, plotted against $N$, the number of cycles. Fatigue strength can be approximated by Equation (10), where $a$ and $d$ are defined by points located at $10^{3}$ and $10^{6}$. 


$$
S_{f}=a N^{d}
$$

Fluctuating stresses typically follow a sinusoidal pattern and include midrange, or mean, stress, $\sigma_{m}$ and amplitude, or alternating stress, $\sigma_{a}$. High cycle fatigue data, defined as the data between $10^{3}$ and $10^{6}$, can be correctly adjusted by taking the logarithmic transform of Equation (10) [43].

Hwang and Han et al. [28] studied the fatigue of composites and their model presented in Equation (11) was found to fit experimental data more closely than log-linear or power law equations (e.g., Basquin's law), where $r$ is the applied stress level, $N$ is the number of cycles, and $b$ and $c$ are material parameters [28].

$$
N=[b(1-r)]^{\frac{1}{c}}
$$

\subsection{Experimental Uncertainty}

In equations, the errors found in the independent variables can propagate to result in an overall error found in the dependent variable. Equation 12 displays the function used to determine experimental uncertainty of stress for this study, where $S$ is stress, $l$ is specimen length, and $h$ is specimen thickness [44].

$$
\delta S=\sqrt{\left(\frac{\partial S}{\partial f} \delta f\right)^{2}+\left(\frac{\partial S}{\partial l} \delta l\right)^{2}+\left(\frac{\partial S}{\partial h} \delta h\right)^{2}}
$$




\section{METHODOLOGY}

The methodology section describes the experimental apparatus and the testing protocols for experimentation. Multiphoton microscopy images were captured from untreated and nicotine-treated murine arteries to visualize elastin and collagen in the arterial wall. Fatigue parameters were extracted from data captured by performing

preconditioning, stress relaxation, and fatigue testing on an additional set of untreated and nicotine-treated murine arteries.

\subsection{Specimen Acquisition}

Murine arteries from BL6/C57 male mice, aged 10-12 weeks, were provided by the research group of Dr. Tsao at the Palo Alto Veterans Institute for Research (PAVIR). Procedures used for animal care and tissue harvesting were approved by the Institutional Animal Care and Use Committee (IACUC) for the Palo Alto Veterans Institute for Research (PAVIR) at the VA Palo Alto Health Care System (VAPAHCS). Although no single model has been shown to recreate human diseases, rat and mice models are shown to be useful tools in the study of cardiovascular disease [45].

The first set of murine arteries contained ten artery specimens harvested from ten mice to capture multiphoton images. Five murine specimens were treated with nicotine through a subcutaneous osmotic mini-pump at a concentration of $25 \mathrm{mg} / \mathrm{kg} /$ day for a total of 28 days, while the second set of five specimens were left untreated. The ten arteries 
used for multiphoton imaging were unstretched, or otherwise did not undergo fatigue testing.

The second set contained ten artery specimens to capture fatigue parameter extraction after undergoing three stages of testing - preconditioning, stress relaxation, and fatigue testing. Five specimens were from mice treated with nicotine through a subcutaneous osmotic mini-pump at a concentration of $25 \mathrm{mg} / \mathrm{kg} /$ day for a total of 28 days, while the second set of five specimens were from untreated mice. For each specimen, the thoracic portion of the aorta was fatigue-tested.

The specimens were chosen based upon availability. The arteries were cut along the axial axis to achieve an open-circumferential configuration for imaging and fatigue testing.

\subsection{Experimental Apparatus}

The microtensometer apparatus for this study used a $113 \mathrm{~g}$ load cell (OMEGA, Norwalk, Connecticut, USA), a programmable motorized nanopositioner (MP-285, Sutter Instrument, Novato, California, USA) and a 24-bit digital acquisition unit (Loadstar DI-1000U, Loadstar, Fremont, California, USA).

A script was written for the nanopositioner prior to execution of testing protocols. The data acquisition system (DAQ) and nanopositioner worked simultaneously to ensure data points were recorded in real time.

The specimen was secured onto aluminum clamps and vertically loaded into a stationary container filled with physiological fluid (Tyrode's solution). The vertical 
configuration used for tensile testing specimens is similarly used by de Gelidi et al. [46] and Washington et al. [29]. Once the specimen was vertically loaded, the load cell was attached to the clamps using a spring loaded pin. The schematic is shown in Figure 1.

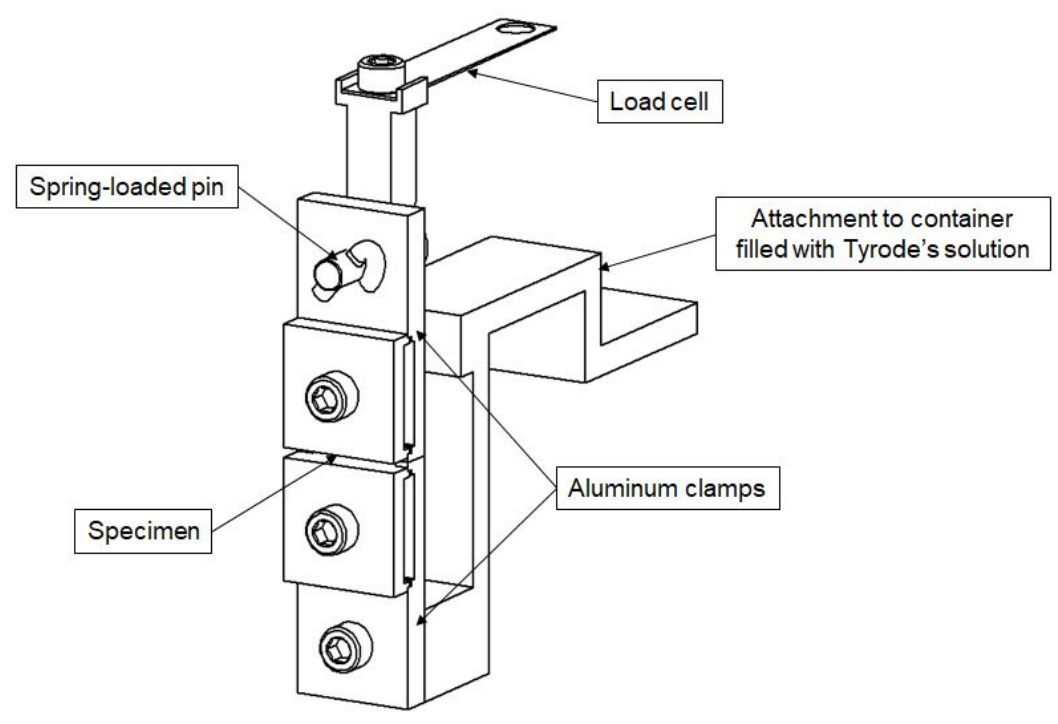

Figure 1. Labeled schematic diagram of specimen set-up.

Each specimen was loaded in a pair of aluminum clamps that are each sized approximately $25 \mathrm{~mm} \times 13 \mathrm{~mm}$, with top and bottom components as shown in Figure 2 . Placement of the artery at the flushed interface of the two clamps allowed for an initial $1.0 \mathrm{~mm}$ gauge length. These clamps were designed to have a step-down to ensure the artery was securely fastened between the top and bottom components. 


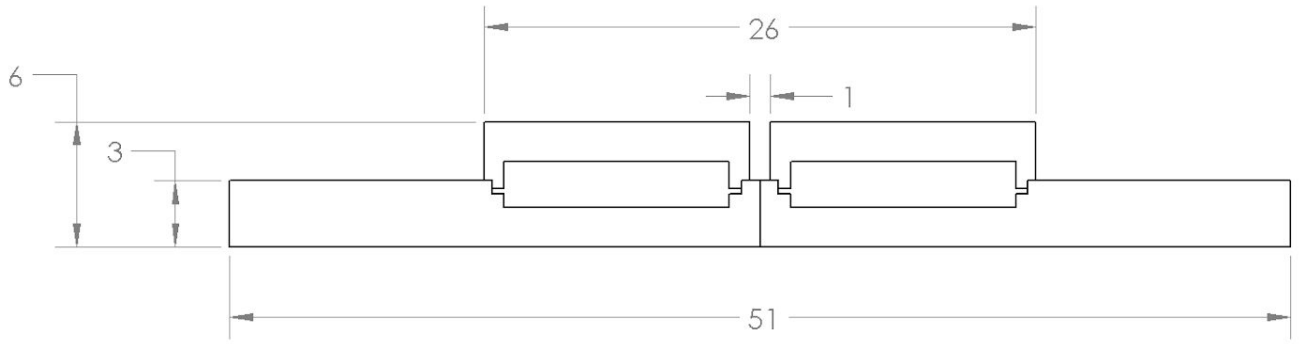

Figure 2. Dimensional drawing of aluminum clamps used to secure the artery. Measurements shown are in millimeters.

The load cell was placed in series with the test specimen, in a similar arrangement to the configuration used by Eng et al. [47] for mechanical testing of soft materials, such as alginate and agarose.. Accordingly, the measured force was based on equivalent stiffness of the tested specimen $k_{1}$ and the load cell $k_{2}$ in series, as shown in Equation (13).

$$
k_{e q}=\frac{k_{1} k_{2}}{k_{1}+k_{2}}
$$

\subsection{Experimentation}

In this work, fatigue is defined as the reduction in resistance to a prescribed extension as the number of extension cycles increases. This definition applies to extensometric testing. Alternatively but not in this work, fatigue can also be defined in terms of tensometric testing, in which a specimen elongates to a greater extent under a prescribed tensile force as the number of loading cycles increases.

\subsubsection{Fatigue Testing Protocol}

This protocol describes the strain-controlled fatigue testing of uniform gage specimens, mimicking testing considerations as in the ASTM E606 standard [48]. Prior 
to mechanical testing, the thickness of each specimen was determined by placing the specimen between two glass slides and using a digital micrometer (Mitutoyo 293-340) with $1 \mu \mathrm{m}$ resolution. To ensure consistent measurement, each specimen was saturated in a physiological fluid (Tyrode's solution) and the fluid was retained around the specimen by a boundary marked by a hydrophobic pen (ImmEdge H-4000, Vector Laboratories, Inc. Burlingame, California, USA).

To account for clamp weight vertically in the physiological fluid, the position of the upper grip was adjusted under microscope to offset the weight of the upper aluminum clamp assembly to re-establish an initial gauge length of $1.0 \mathrm{~mm}$. The gap between the vertically-loaded opposing clamps was inspected under microscope and adjusted by no more than $40 \mu \mathrm{m}$ to ensure that there was no metal-to-metal contact. Each specimen was then placed between the clamps, shown in Figure 1. For consistent clamping, the screws on the clamps were secured with $71 \mathrm{~N} \cdot \mathrm{mm}(10 \mathrm{in} \cdot \mathrm{oz})$ of torque.

Three stages of testing were conducted: (1) a slow series of five pre-loading and unloading cycles, by applying a strain of $100 \%$ (actuator extension to $1000 \mu \mathrm{m}$ ) at a rate of $200 \mu \mathrm{m} / \mathrm{s}$, and returning to gauge length at the same speed; (2) one rapid step change at a rate of $5000 \mu \mathrm{m} / \mathrm{s}$ to a strain of $100 \%$, with a hold at constant strain for $30 \mathrm{~s}$ in order to measure stress relaxation; and (3) 500 cycles of repetitive loading with a mean strain of $150 \%$ and a superimposed alternating strain of $\pm 50 \%$ (i.e., actuator extension between $1000 \mu \mathrm{m}$ and $2000 \mu \mathrm{m})$. The actuator speed was set to $5000 \mu \mathrm{m} / \mathrm{s}$ for fatigue loading, but actual speed was subjected to acceleration and deceleration at each reversal, such that the 
typical time to complete 500 cycles was approximately 12 minutes. A similar protocol was performed by Remache et al. [13], which included multiple tests, such as cyclic tensile test, strain rate test, and stress relaxation tests.

Force measurements for the transient response during stress relaxation were recorded at a sampling rate of $100 \mathrm{~Hz}$. Force measurements for preconditioning and fatigue cycling were recorded at a sampling rate of $10 \mathrm{~Hz}$. The frequency rates ensure that even at maximum speed of $5000 \mu \mathrm{m} / \mathrm{s}$ over a travel distance of $1000 \mu \mathrm{m}$, the Nyquist-Shannon sampling criterion is satisfied. In other words, the sampling interval of $0.1 \mathrm{~s}$ is half of the fastest possible (one-directional) actuation time of $0.2 \mathrm{~s}$. In order to measure the width of each sample, and to observe the extent of necking deformation during extension, microscope camera images were captured throughout preconditioning and stress relaxation with one image captured every $2 \mathrm{~s}$, and fatigue testing with one image captured every $10 \mathrm{~s}$. These images also served the benefit of inspecting for defects such as artery side-branch holes or local tears, if any.

The set of slow extension cycles provided initial nonlinear stiffness and a measure of hysteresis, quantified as loss in strain energy. The stress relaxation stage of measurement provided a measure of viscoelastic response in terms of a time constant. The fatigue testing stage provided four characteristic parameters, as described in Section 4.4.

\subsubsection{Multiphoton Microscopy Protocol}

Multiphoton images of unstretched specimens were captured using an upright microscope (Leica DM6000, Buffalo Grove, Illinois, USA). Specimens were placed on 
glass slides, and underwent water immersion microscopy. A z-stack was generated for each artery with images sliced at every $2 \mu \mathrm{m}$. Within the z-stack, elastin and collagen fibers signals were captured by detection of 2PEF and SHG, respectively [6]. Each specimen was imaged at two different locations.

\subsection{Fatigue Model Fitting}

Fatigue life of materials is typically represented by a log-linear plot, which graphs stress-to-failure to number of cycles. Although the log-linear fits plot cycles to failure, this study observed the fatigue profile during cyclic testing. The fatigue model used in this study is adapted from the model presented by Hwang and Han et al. [28], which is a fatigue model that determines the number of cycles to failure from normalized applied stress level. Rearranging Equation (11) results in Equation (14), where $r$ is the normalized applied stress level, $N$ is the number of cycles, and $b$ and $c$ are material parameters.

$$
r=1-\frac{N^{c}}{b}
$$

The fatigue model that is used in this study outputs peak stress rather than normalized applied stress level. Equation (14) was used to extract parameters $b$ and $c$ for each fatigue plot when the stresses were normalized to 1 . To generate an equation that would model a non-normalized fatigue plot, a parameter associated with the maximum stress needed to be multiplied to Equation (14) to shift the value of 1 to the value of the maximum stress. 
Thus, the equation used to plot against the fatigue data is shown in Equation (15), where $S$ is the stress; $N$ is the number of cycles; and $A, B$, and $C$ are material parameters.

$$
S=A-B N^{C}
$$

A fourth parameter, $D$, describes the width of the fatigue data, or the difference between the maximum and minimum stresses as the data reached a plateau. Parameters $A, B$, and $C$ were found using the $c f t o o l$ function in MATLAB. Parameter $D$ was calculated at approximately 400 cycles for all ten specimens.

\subsection{Morphometric Parameter Extraction}

In this protocol, elastin multiphoton images were extracted from each z-stack by exporting .tif images from .lif files using LAS X software (Leica Microsystems, Buffalo Grove, Illinois, USA) to be analyzed. Images of collagen were not extracted due to lack of visibility of collagen fibers within the z-stack .lif file. However, the observation of elastin fibers under exposure to nicotine does not lack merit. Studies have shown that treatments of glycation and nicotine correlate with arterial stiffening [29], [40]. Additionally, it has been shown that glycation of elastin alters the structure of the elastin network [49]. Elastin fibers are also associated with maintaining resistive properties and are shown to degrade when exposed to nicotine [17], [36].

Red channels were adjusted to increase intensity in the .lif file so that the elastin can be better visualized. The multiphoton images were converted to black and white images with a custom script developed in MATLAB, equipped with the Image Processing Toolbox. A series of MATLAB functions were executed on one image from two 
locations per specimen. The image was dictated by the depth at $50 \%$ of each z-stack to average parametric values across the two tif images for each untreated and unstretched specimen, as well as each nicotine-treated and unstretched specimen.

The methodology to transform elastin images to skeletonized images followed closely to the steps performed by Koch et al. [33]. Each image was selected using the dialog box (uigetdir) and converted from a truecolor (RGB) image to a grayscale image (rgb2gray). Once in grayscale, the image was subjected to 2-D median filtering (medfilt2), enhancement of the contrast using contrast-limited adaptive histogram equalization (adapthisteq), and saturation of the bottom and top 1\% of pixels (imadjust). A global threshold from the image was computed (graythresh) and multiplied by a factor of 1.35 to reduce the number of white pixels when converting to a binary image (imbinarize). After converting the image to a black and white image, a series of morphological operations were performed to prepare the image for skeletonization. Isolated pixels were removed (bwmorph( 'clean')), the image was dilated (structuring element $(\mathrm{SE})$ disk radius $=1$, imdi late), and then eroded (SE disk radius $=1$, imerode). 8-connected pixels were eliminated (bwmorph( 'diag')) to improve the connectivity of pixels across the image. Pixels that formed objects smaller than 15 pixels were removed (bwareaopen) and then the images were automatically saved to a separate directory (imwrite).

For skeletonization and morphometric parameter extraction, binary images were analyzed with the DiameterJ plug-in of the Fiji software suite [50]. Morphometric 
parameters mean diameter, standard deviation (SD) of diameter, characteristic length, standard deviation of characteristic length, and intersection density were extracted and compared for unstretched untreated and unstretched nicotine-treated specimens. 


\section{RESULTS AND DISCUSSION}

One major type of results in this thesis is the comparison of experimental fatigue data of untreated and nicotine-treated artery specimens. Average strain energy density values for the first loading of preconditioning testing were calculated, and power law parameters were extracted for stress relaxation curves. Parameters for fatigue data were extracted using a modified power law for each specimen. In addition, morphometric parameters of elastin were extracted from multiphoton microscopy images and compared for unstretched untreated and unstretched nicotine-treated specimens.

\subsection{Untreated and Nicotine-Treated Fatigue Protocol Data}

Section 5.1 presents the preconditioning data captured from the untreated and nicotine-treated specimens. This section also includes the average strain energy density across untreated and nicotine-treated specimens for the first initial loading.

\subsubsection{Preconditioning}

Preconditioning cycles for the untreated and nicotine-treated specimens are shown in Figure 3 and Figure 4, respectively. 
(a)

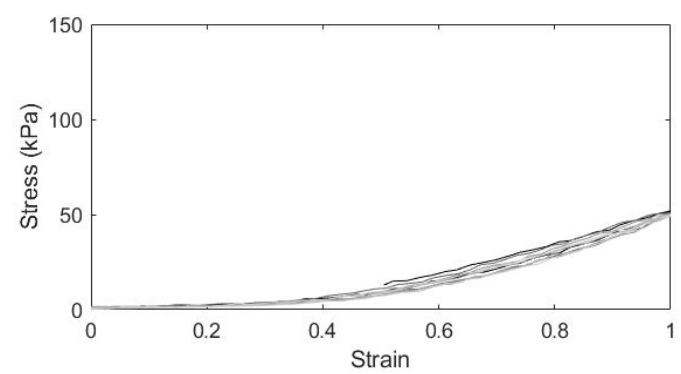

(c)

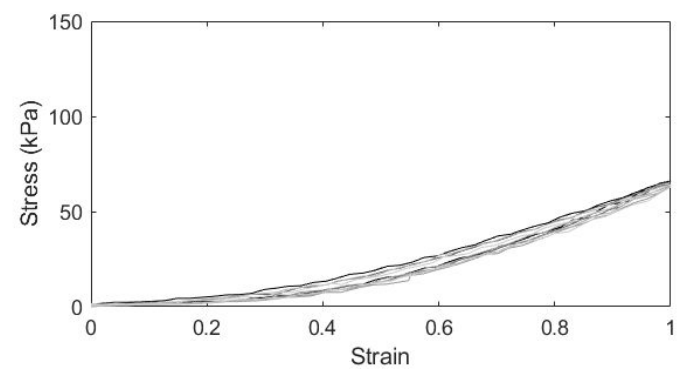

(e)

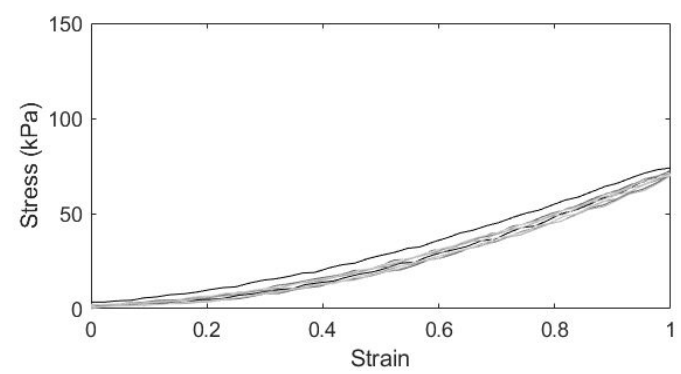

(b)

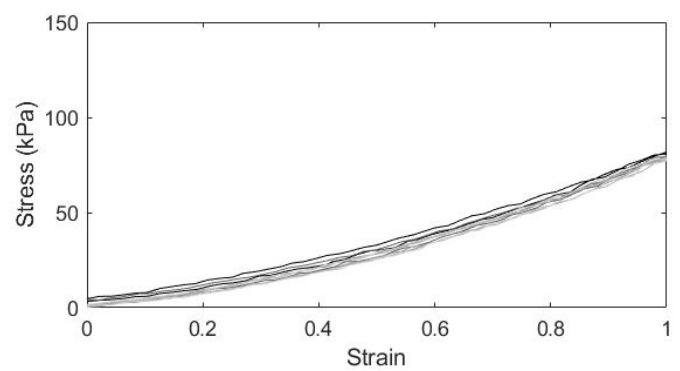

(d)

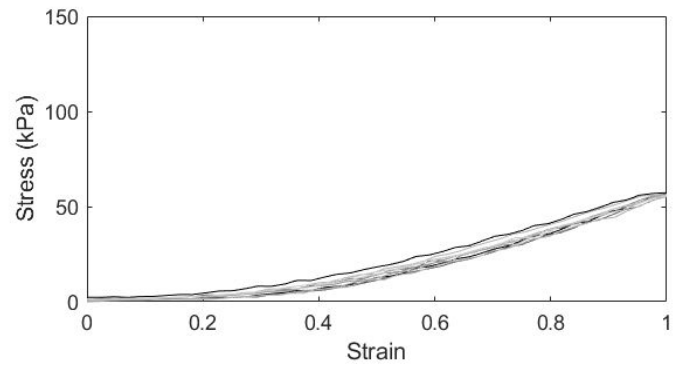

Figure 3. Preconditioning plots for untreated murine arteries. 
(a)

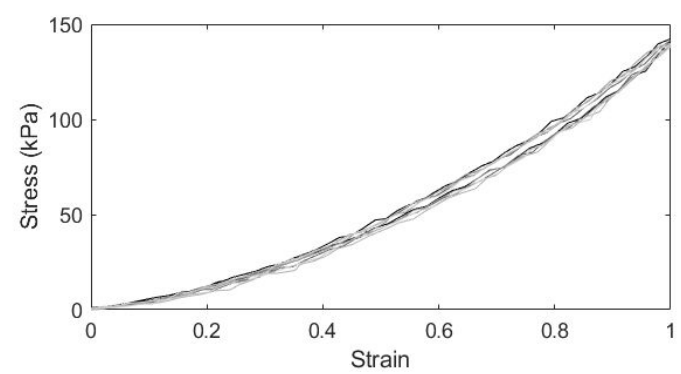

(c)

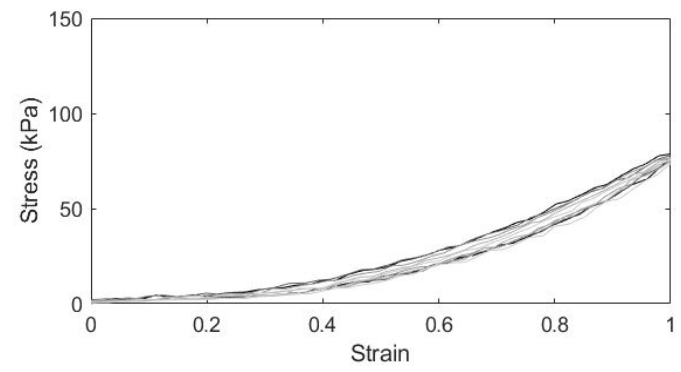

(e)

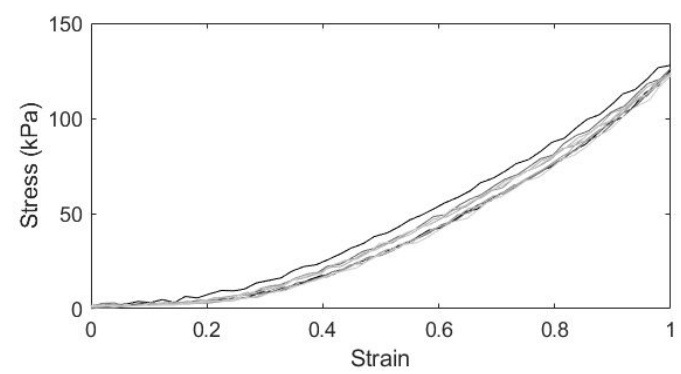

(b)

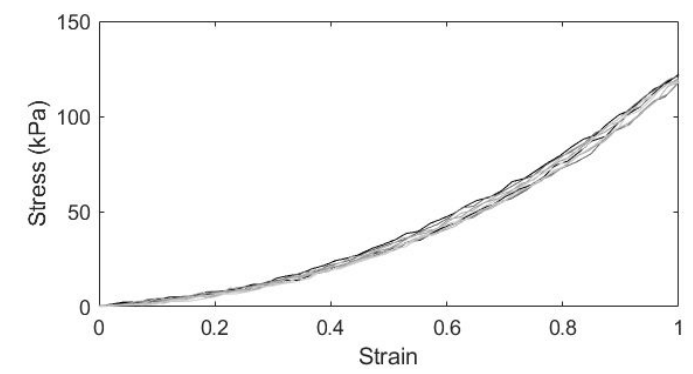

(d)

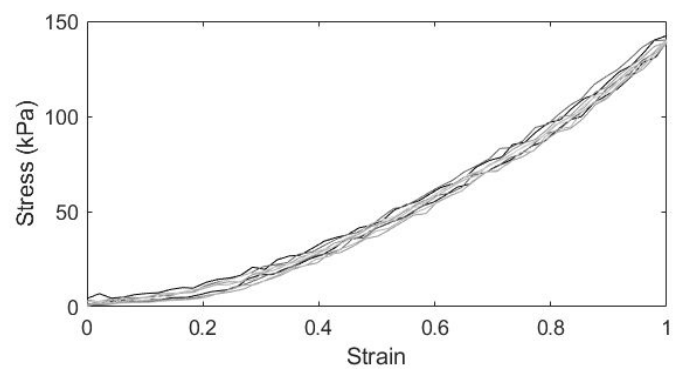

Figure 4. Preconditioning plots for nicotine-treated murine arteries.

Preconditioning was performed prior to subsequent testing to reduce hysteresis and deformation history of the specimen. The graphs present stresses as measured from the load cell for the programmed strain. As the load cell and specimen represent springs in series, a percentage of strain uncertainty due to the load cell deformation under 1.0 strain is discussed in Section 5.3. The strain energy density from the first loading curves of each cycle for the untreated and nicotine-treated specimens was calculated. Strain energy density of the first loading cycles of untreated specimens averaged $30.1 \mathrm{kPa}$, while the 
strain energy density of the first loading cycles of nicotine treated specimens averaged $41.4 \mathrm{kPa}$.

\subsubsection{Stress Relaxation}

Stress relaxation plots were generated for the untreated thoracic specimens and are shown in Figure 5. Stress relaxation plots were generated for the nicotine-treated thoracic specimens and are shown in Figure 6.

(a)

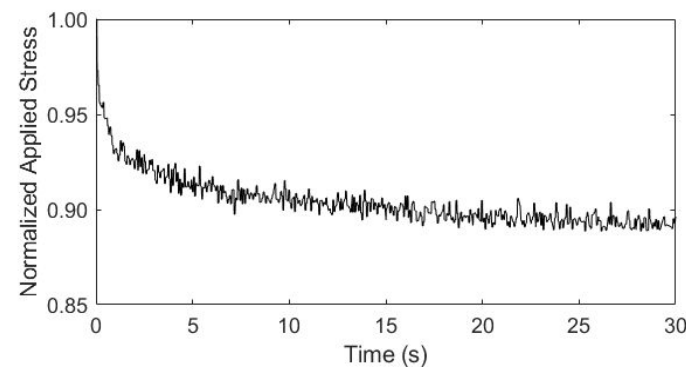

(c)

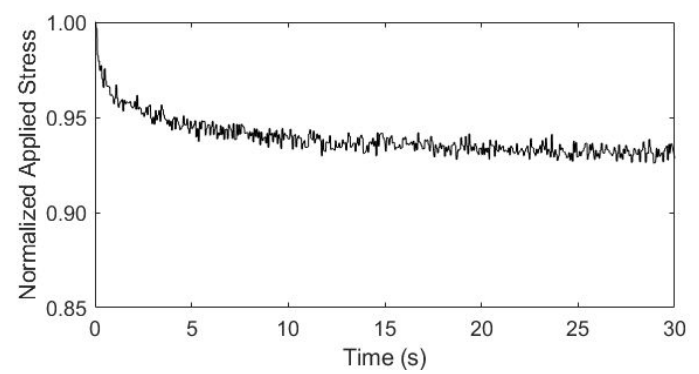

(e)

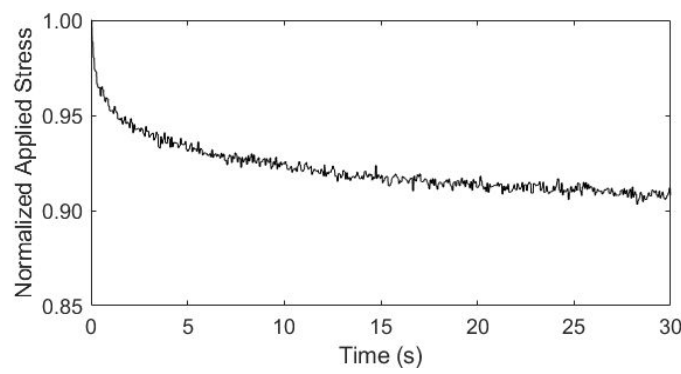

(b)

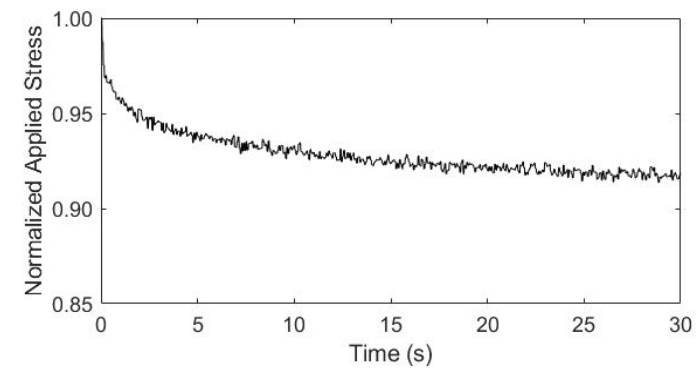

(d)

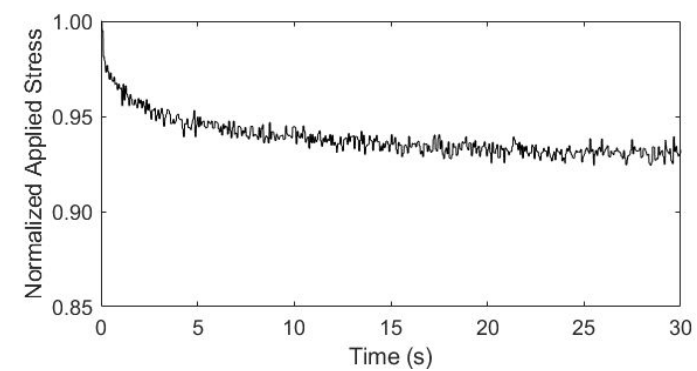

Figure 5. Stress relaxation plots for untreated thoracic arteries. 
(a)

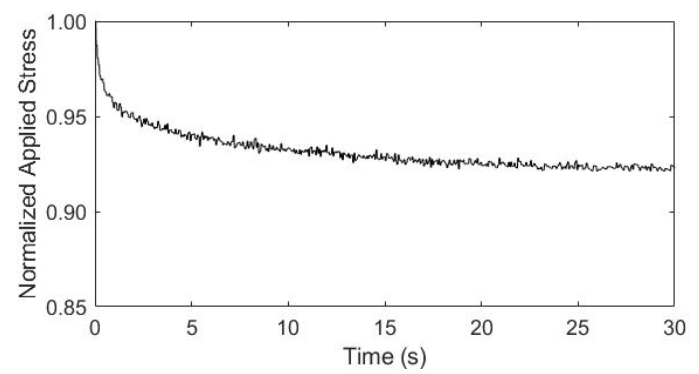

(c)

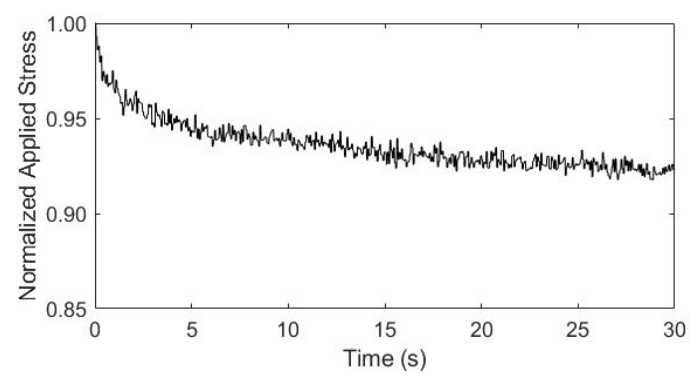

(e)

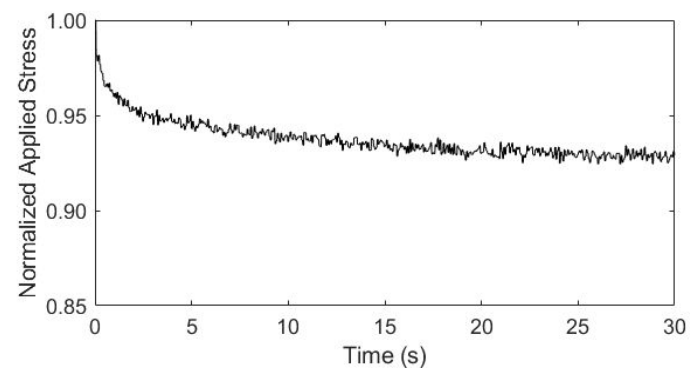

(b)

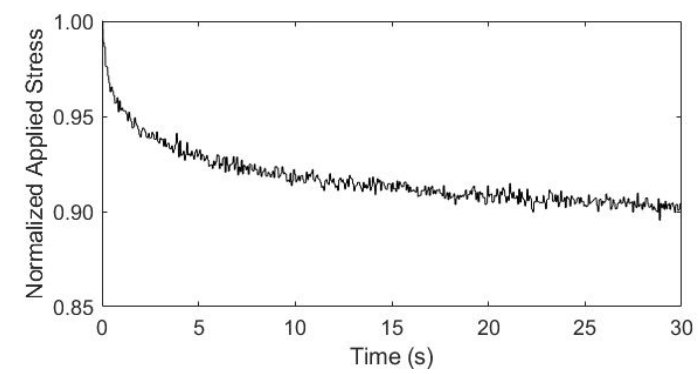

(d)

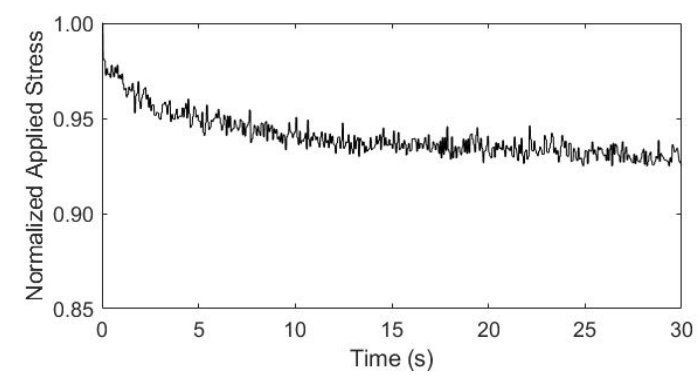

Figure 6. Stress relaxation plots for nicotine-treated thoracic arteries.

Stress was normalized to peak stress and the plots were fitted with the power law shown in Equation (16) using the MATLAB Curve Fitting Toolbox. For this fit, $x$ represents time in seconds, $y$ represents normalized applied stress (e.g., $\mathrm{kPa} / \mathrm{kPa}), b_{1}$ is a dimensionless exponent. For dimensional consistency, the scaling coefficient $a_{1}$ necessarily has units of seconds to the power $\left(1 / b_{1}\right)$.

$$
y=a_{1} x^{b_{1}}
$$


The corresponding stress relaxation plots with fitted lines for five untreated thoracic arteries are shown in Figure 7. The corresponding stress relaxation plots with fitted lines for five nicotine-treated thoracic arteries are shown in Figure 8.

(a)

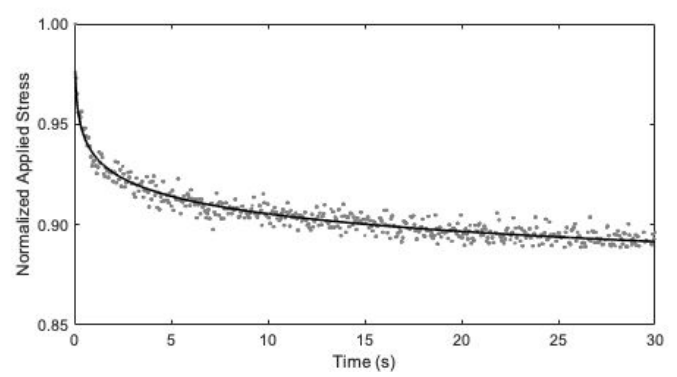

(c)

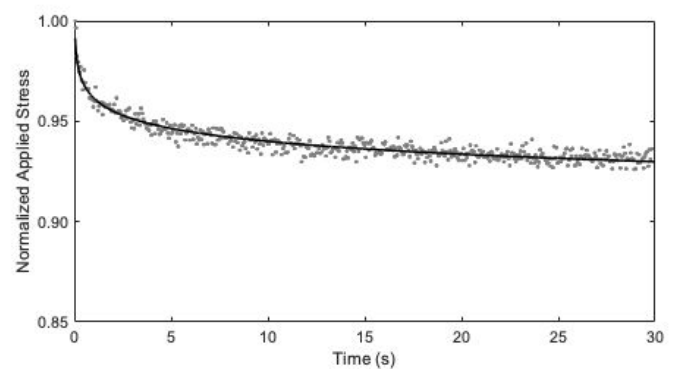

(e)

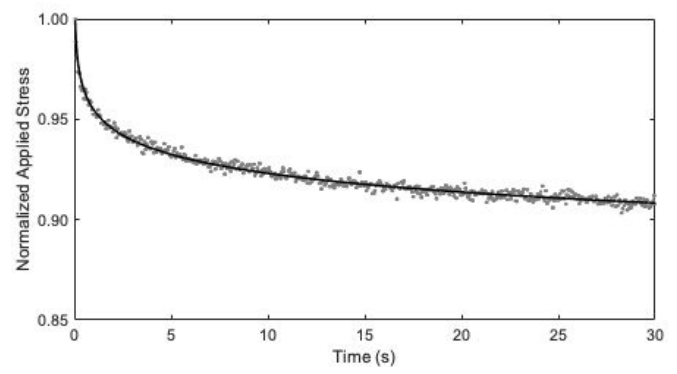

(b)

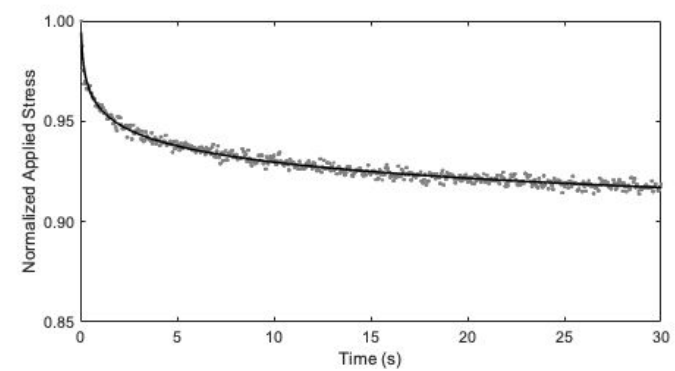

(d)

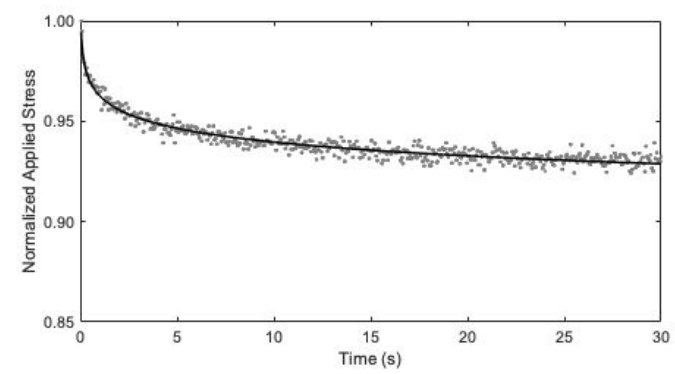

Figure 7. Stress relaxation plots with fitted lines for untreated thoracic arteries. 
(a)

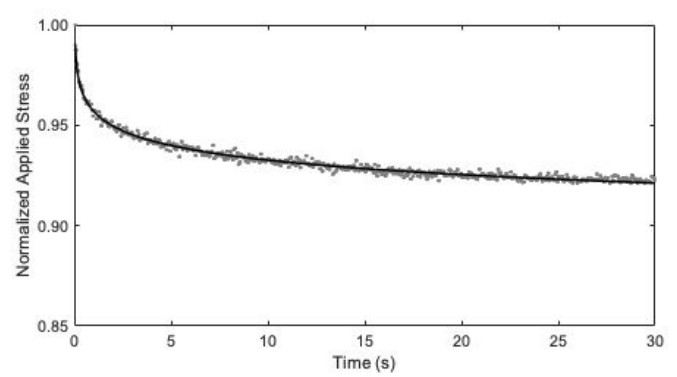

(c)

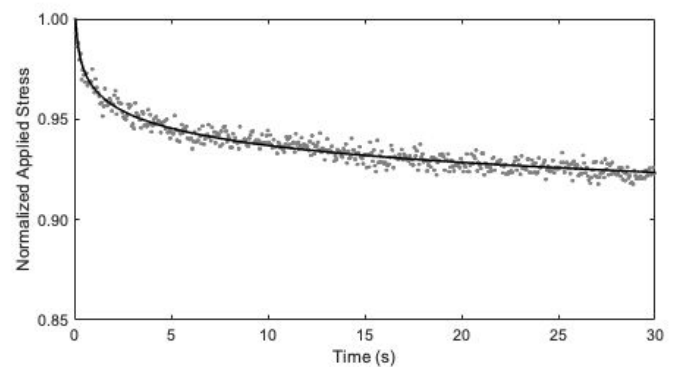

(e)

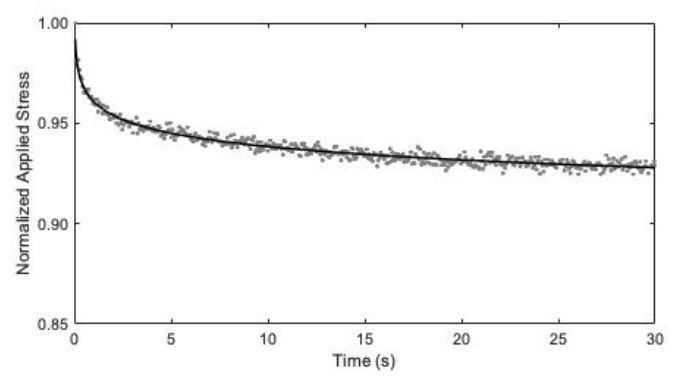

(b)

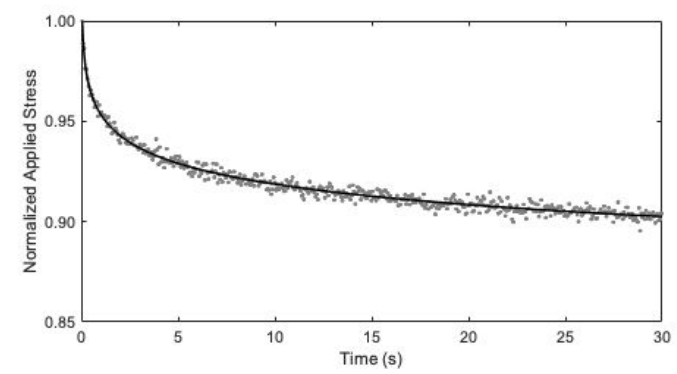

(d)

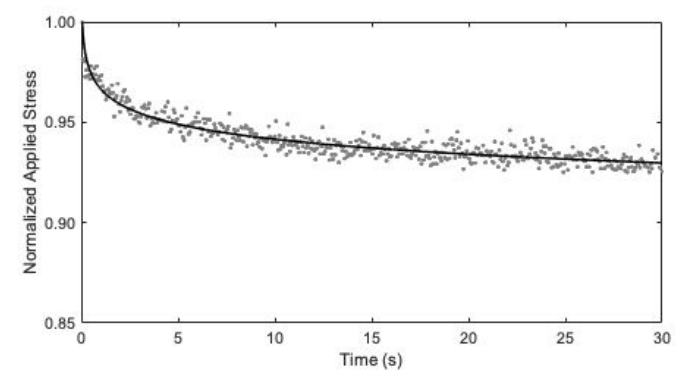

Figure 8. Stress relaxation plots with fitted lines for nicotine-treated thoracic arteries.

Power law constants for untreated specimens are listed in Table 1. Power law constants for nicotine-treated specimens are listed in Table 2.

Table 1. Power Law Constants for Untreated Specimens.

\begin{tabular}{|c|c|c|}
\hline Specimen & $a_{l}$ & $b_{l}$ \\
\hline Untreated Thoracic 1 & 0.935 & -0.0140 \\
\hline Untreated Thoracic 2 & 0.957 & -0.0125 \\
\hline Untreated Thoracic 3 & 0.962 & -0.0099 \\
\hline Untreated Thoracic 4 & 0.963 & -0.0105 \\
\hline Untreated Thoracic 5 & 0.955 & -0.0147 \\
\hline
\end{tabular}


Table 2. Power Law Constants for Nicotine-Treated Specimens.

\begin{tabular}{|c|c|c|}
\hline Specimen & $a_{l}$ & $b_{l}$ \\
\hline Nicotine-Treated Thoracic 1 & 0.957 & -0.0112 \\
\hline Nicotine-Treated Thoracic 2 & 0.954 & -0.0162 \\
\hline Nicotine-Treated Thoracic 3 & 0.966 & -0.0131 \\
\hline Nicotine-Treated Thoracic 4 & 0.967 & -0.0115 \\
\hline Nicotine-Treated Thoracic 5 & 0.961 & -0.0103 \\
\hline
\end{tabular}

A t-test for the difference between two means was performed on parameters $a_{1}$ and $b_{1}$ assuming unequal variances using an alpha value of $\alpha=0.05$. At this significance level there was insufficient evidence to conclude a difference between the untreated and nicotine-treated groups for parameter $a_{1}$ or parameter $b_{1}$. During stress relaxation of the specimen, as a constant strain of 1.0 was held, creep was identified due to the tension of the load cell on the specimen.

\subsubsection{Fatigue Data}

Fatigue data for five untreated murine specimens are shown in Figure 9. Fatigue data for five nicotine-treated murine specimens are shown in Figure 10. 
(a)

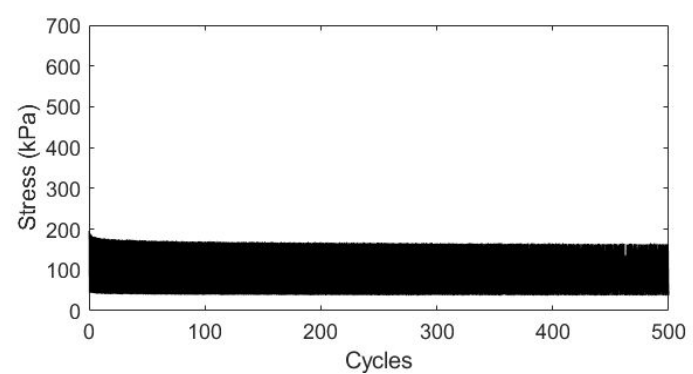

(c)

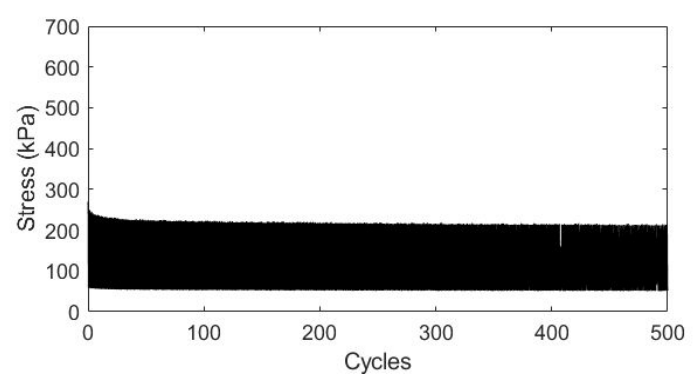

(e)

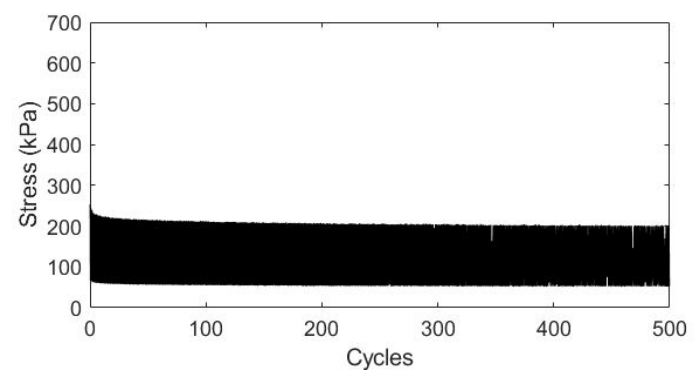

(b)

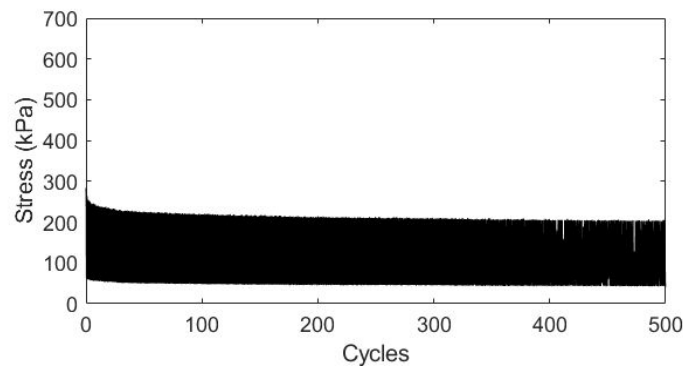

(d)

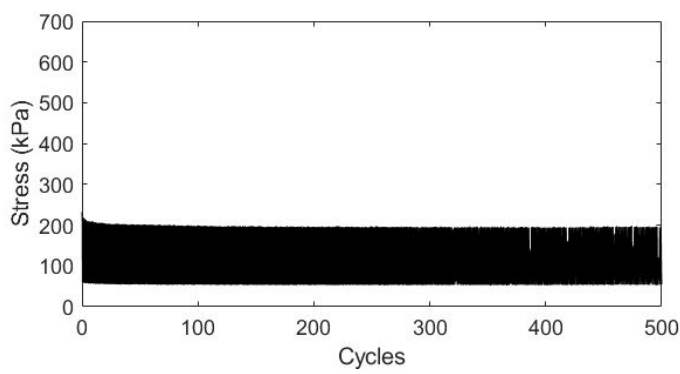

Figure 9. Fatigue data for untreated specimens. 
(a)

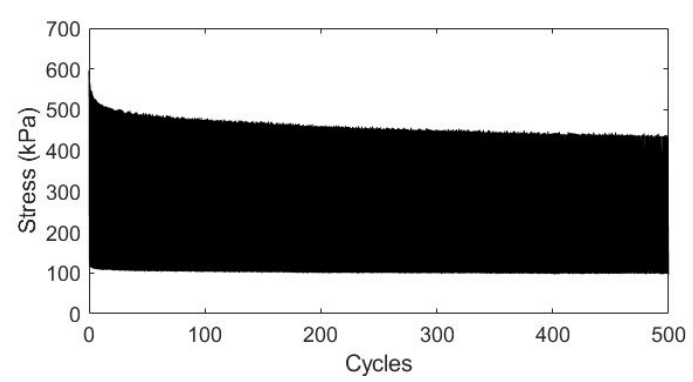

(c)

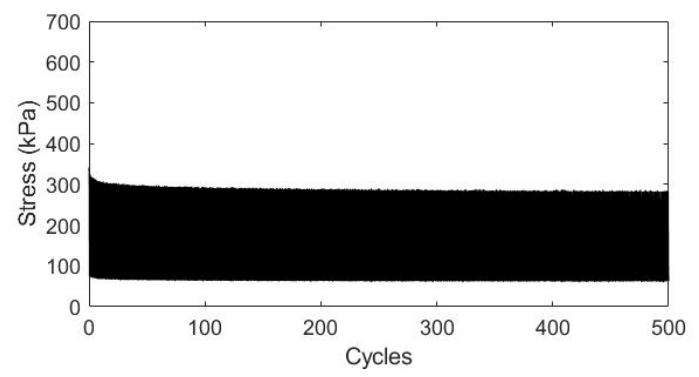

(e)

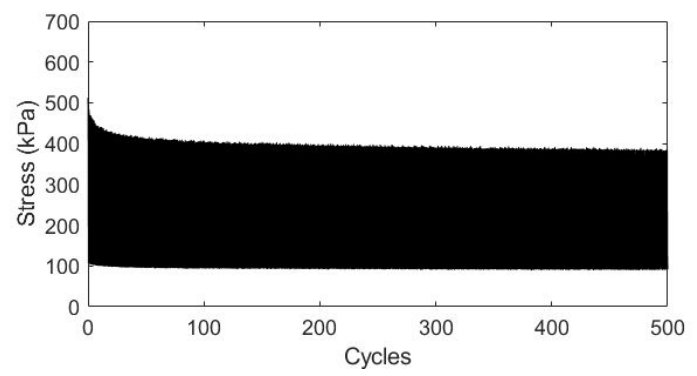

(b)

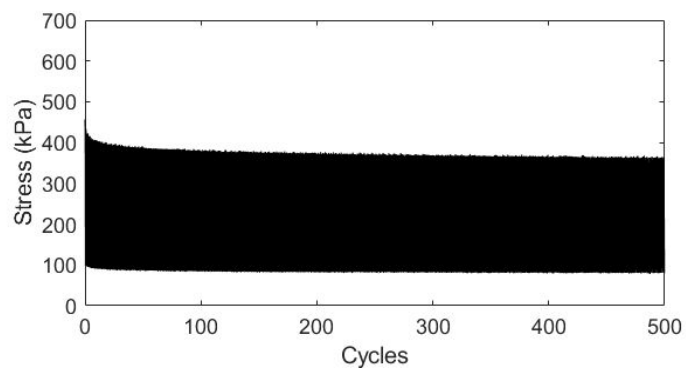

(d)

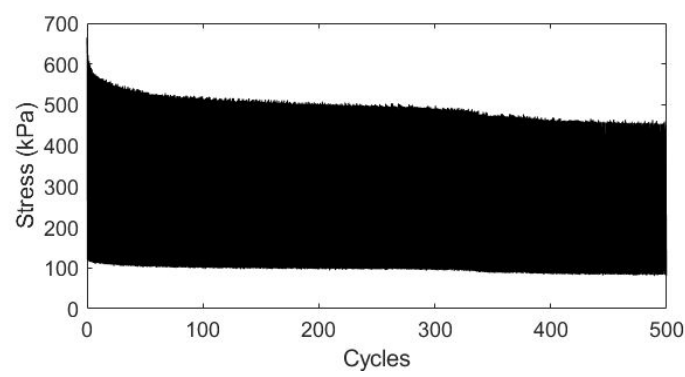

Figure 10. Fatigue data for nicotine-treated specimens.

Parameters $A, B$, and $C$ were extracted from Equation (15) using the curve fitting toolbox in MATLAB, while parameter $D$ was manually calculated. The fatigue parameters are listed in Table 3 for untreated specimens and Table 4 for nicotine-treated specimens. 
Table 3. Parameters $A, B, C$, and $D$ Extracted for Untreated Specimens.

\begin{tabular}{|c|c|c|c|c|}
\hline Specimen & $A$ & $B$ & $C$ & $D$ \\
\hline Untreated Thoracic 1 & 196 & 87.1 & 0.021 & 64.1 \\
\hline Untreated Thoracic 2 & 284 & 139 & 0.032 & 82.5 \\
\hline Untreated Thoracic 3 & 269 & 132 & 0.015 & 82.4 \\
\hline Untreated Thoracic 4 & 232 & 104 & 0.017 & 70.9 \\
\hline Untreated Thoracic 5 & 253 & 111 & 0.028 & 74.8 \\
\hline
\end{tabular}

Table 4. Parameters $A, B, C$, and $D$ Extracted for Nicotine-Treated Specimens.

\begin{tabular}{|c|c|c|c|c|}
\hline Specimen & $A$ & $B$ & $C$ & $D$ \\
\hline Nicotine-Treated Thoracic 1 & 596 & 295 & 0.027 & 175 \\
\hline Nicotine-Treated Thoracic 2 & 457 & 212 & 0.023 & 145 \\
\hline Nicotine-Treated Thoracic 3 & 341 & 156 & 0.020 & 113 \\
\hline Nicotine-Treated Thoracic 4 & 665 & 333 & 0.034 & 192 \\
\hline Nicotine-Treated Thoracic 5 & 512 & 255 & 0.021 & 151 \\
\hline
\end{tabular}

Fatigue parameters presented in this study are taken from the model shown in Equation (15) and are plotted in Figure 11 to compare untreated and nicotine-treated parameters. 
(a)

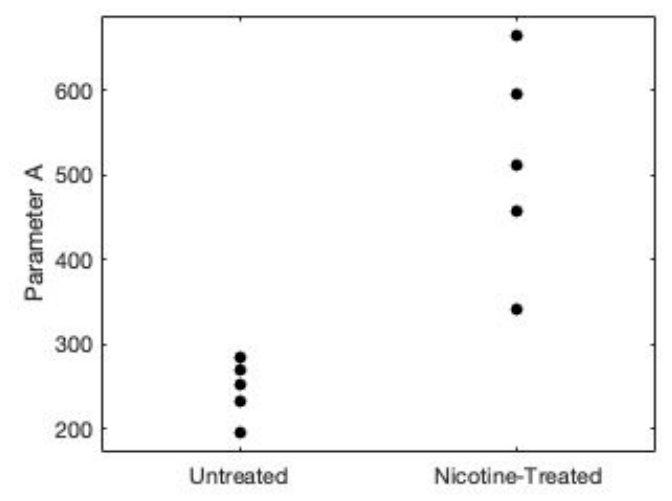

(c)

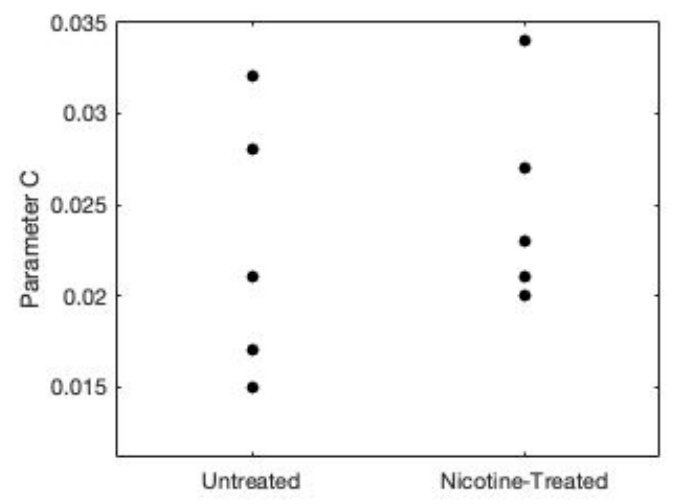

(b)

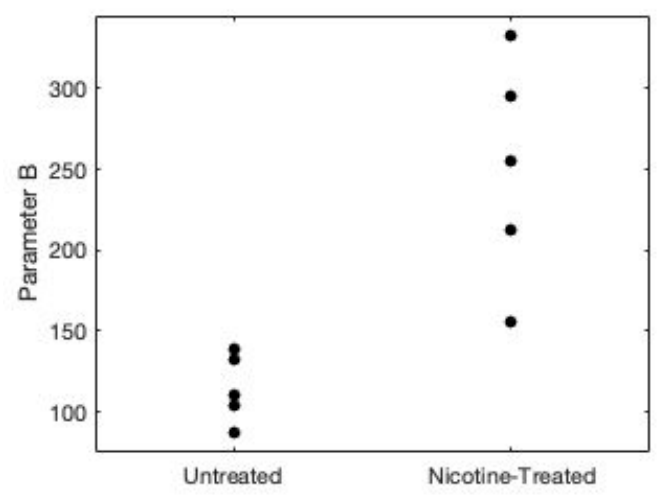

(d)

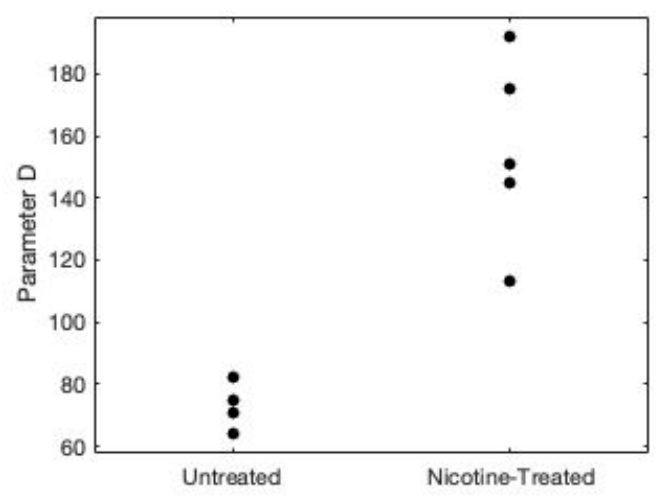

Figure 11. Scatter plots of parameters extracted from fatigue model for untreated and nicotine-treated specimens.

The fatigue data were plotted with Equation (15) using the extracted parameters, shown in Figure 12 for fatigue-tested untreated specimens and Figure 13 for fatigue-tested treated specimens. Parameter $D$ is not plotted as it is does not contribute to the fitted line. 
(a)

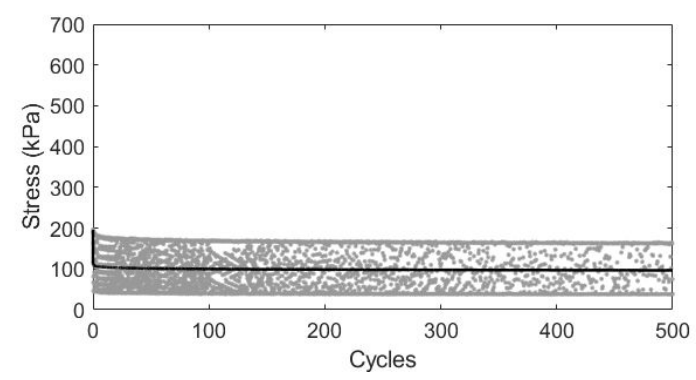

(c)

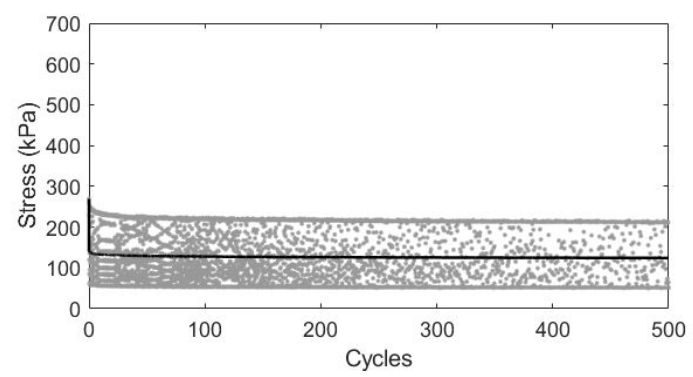

(e)

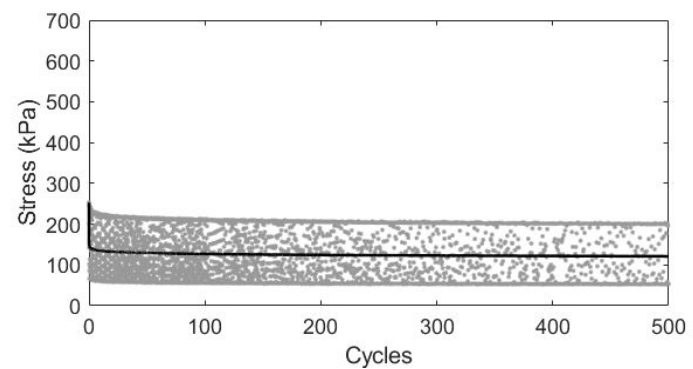

(b)

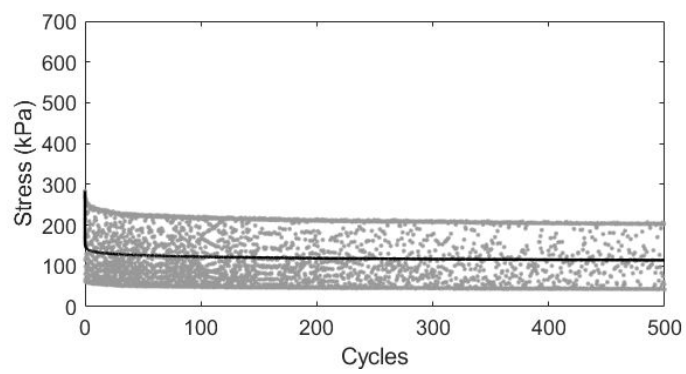

(d)

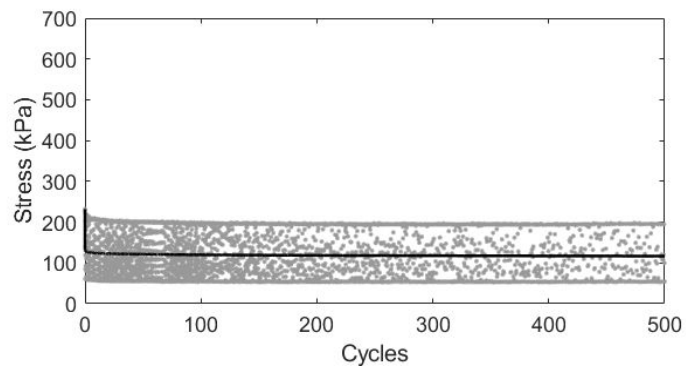

Figure 12. Fatigue data with fitted lines for untreated thoracic arteries. 
(a)

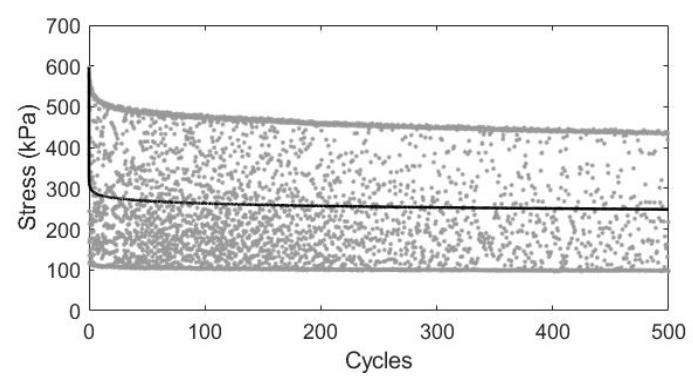

(c)

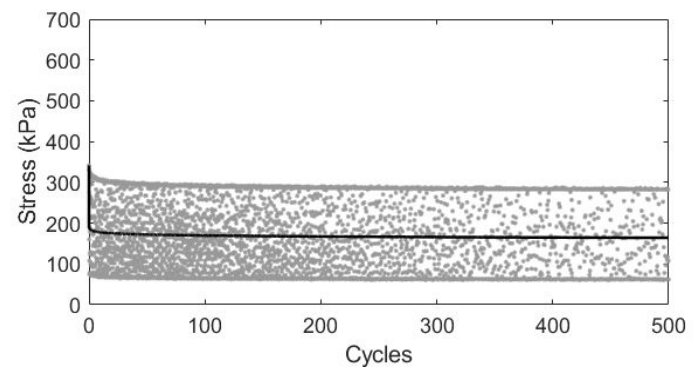

(e)

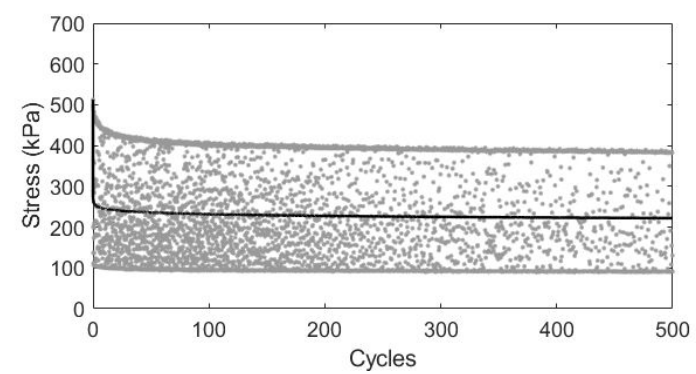

(b)

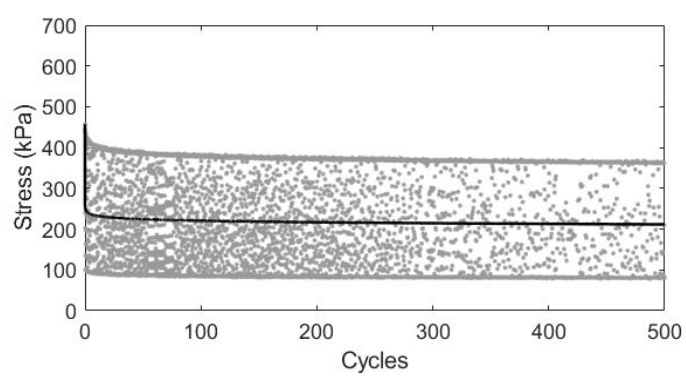

(d)

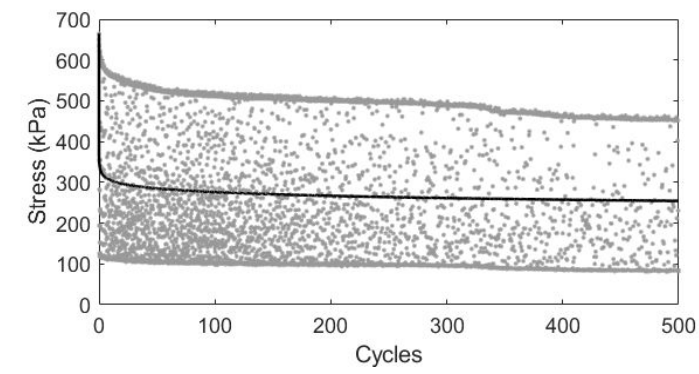

(f)

Figure 13. Fatigue data with fitted lines for nicotine-treated thoracic arteries.

Parameters $A, B, C$, and $D$ can be described as the peak stress of the cyclic data, loss of tension, degradation slope across cycle count, and width of oscillation band, respectively. To better understand the effects of the parameter values, Figure 14 shows a simulated fatigue output plot set to 300 cycles, while Figure 15 displays the effects to the simulated fatigue output plot when each parameter is independently increased. The effects of increasing parameters $A, B, C$, and $D$ are shown in Figure 15(a), 15(b), 15(c), and 15(d), respectively. 


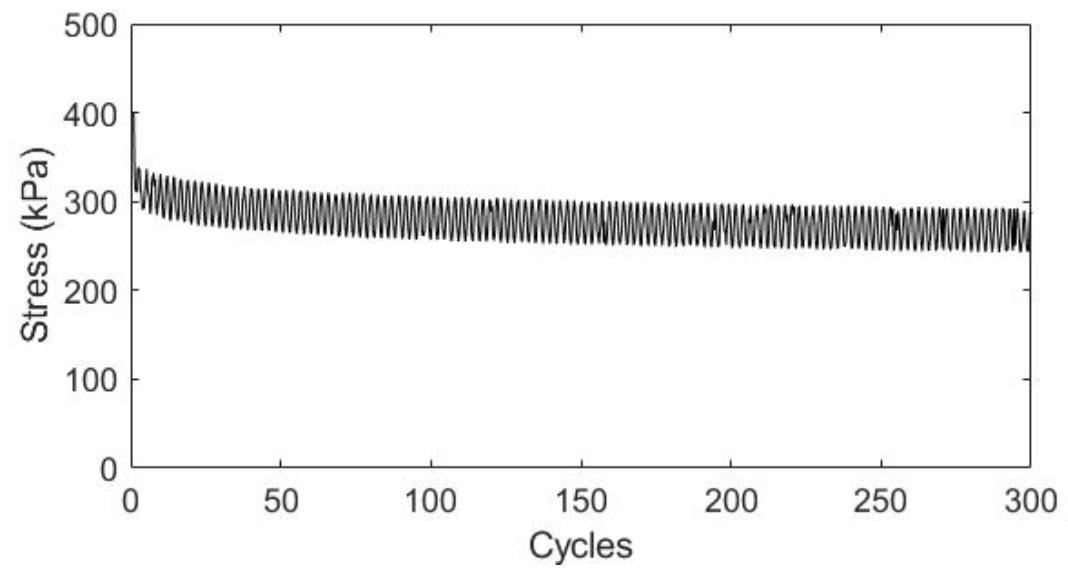

Figure 14. Simulated model of fatigue output.

(a)

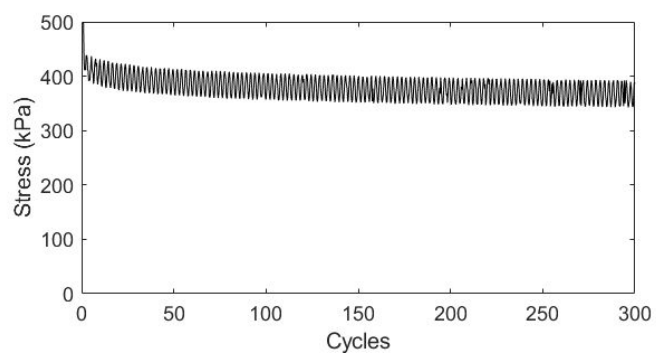

(c)

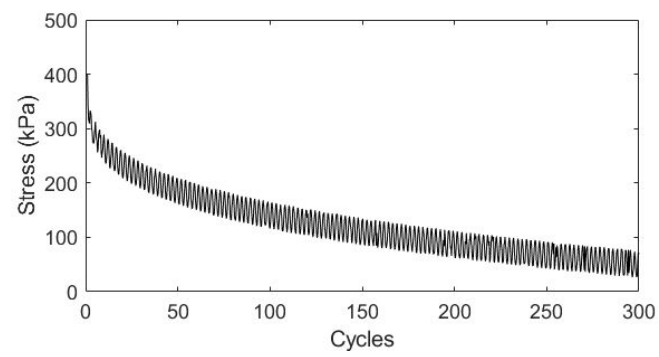

(b)

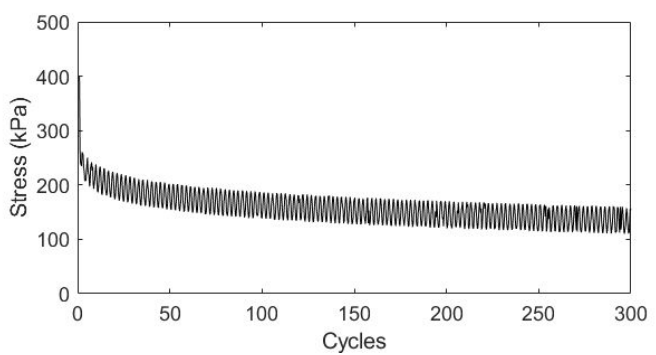

(d)

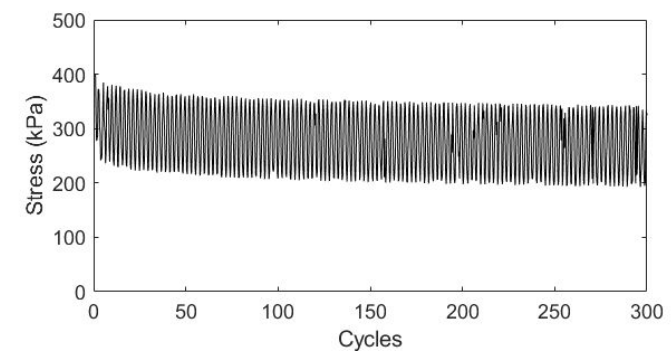

Figure 15. Effects on simulated model of fatigue after an increase in value of individual parameters $A(\mathrm{a}), B(\mathrm{~b}), C(\mathrm{c})$, and $D(\mathrm{~d})$.

As parameter $A$ increases, the amplitude of the entire plot increases. As parameter $B$ increases, the peak stress does not change, but the body of the fatigue data shifts downward while maintaining similar slope to the original plot. As parameter $C$ increases, 
the slope of the fatigue plot declines to a lower stress value as cycles increase. As parameter $D$ increases, the oscillation band increases in width. Table 3 displays the average values of parameters $A, B, C$, and $D$ found for untreated and nicotine-treated specimens, as well as the percent differences between the averages in reference to untreated specimens.

Table 5. Average Values of Fatigue Parameters for Untreated and Nicotine-Treated Specimens.

\begin{tabular}{|c|c|c|c|c|}
\hline \multicolumn{5}{|c|}{ Average Fatigue Parameter Values } \\
\hline Specimen & $A$ & $B$ & $C$ & $D$ \\
\hline Untreated & 246.7 & 114.5 & 0.0226 & 74.93 \\
\hline Nicotine-Treated & 514.3 & 250.2 & 0.0250 & 155.1 \\
\hline$\%$ Difference from Untreated & $+108.46 \%$ & $+118.44 \%$ & $+10.44 \%$ & $+107.03 \%$ \\
\hline
\end{tabular}

Nicotine-treated specimens exhibited an $108.46 \%$ higher value of parameter $A$, $118.44 \%$ higher value of parameter $B, 10.44 \%$ higher value of parameter $C$, and $107.03 \%$ higher value of parameter $D$. Static loading typically outputs a maximum stress value, which is associated with parameter $A$, whereas fatigue loading generates additional parameters $B, C$, and $D$. Statistical t-tests assuming unequal variances were performed between untreated and nicotine-treated parameters for each of the fatigue parameters. With an alpha value of 0.05 , significance was found between untreated and nicotine-treated groups for parameters $A, B$, and $D$. The difference in parameter $A$ showed significance with a $p$-value of 0.006 , parameter $B$ showed a significance with a $p$-value of 0.009 , and parameter $D$ showed significance with a $p$-value of 0.002 . The average value for parameter $A$ for both untreated and nicotine-treated specimens align with previous studies, which conclude that nicotine-treated specimens exhibit greater stiffness, or 
higher stress values, than untreated specimens [36], [37], [40]. However, fatigue loading generates additional parameters that characterize the fatigue output plot, indicating that fatigue testing may reveal changes to the mechanical behavior of murine arteries that is undetectable under static loading. The fatigue data can be linearized to perform statistical analysis in accordance to ASTM E739 [51].

\subsection{Multiphoton Image Analysis}

Leica LAS X software was used to analyze the z-stack images of each specimen artery specimen allocated for multiphoton imaging. This protocol focuses on the comparison of the structure of elastin of nicotine-treated and untreated. Sample images of elastin extracted from the z-stacks of untreated specimens are shown in Figure 16.
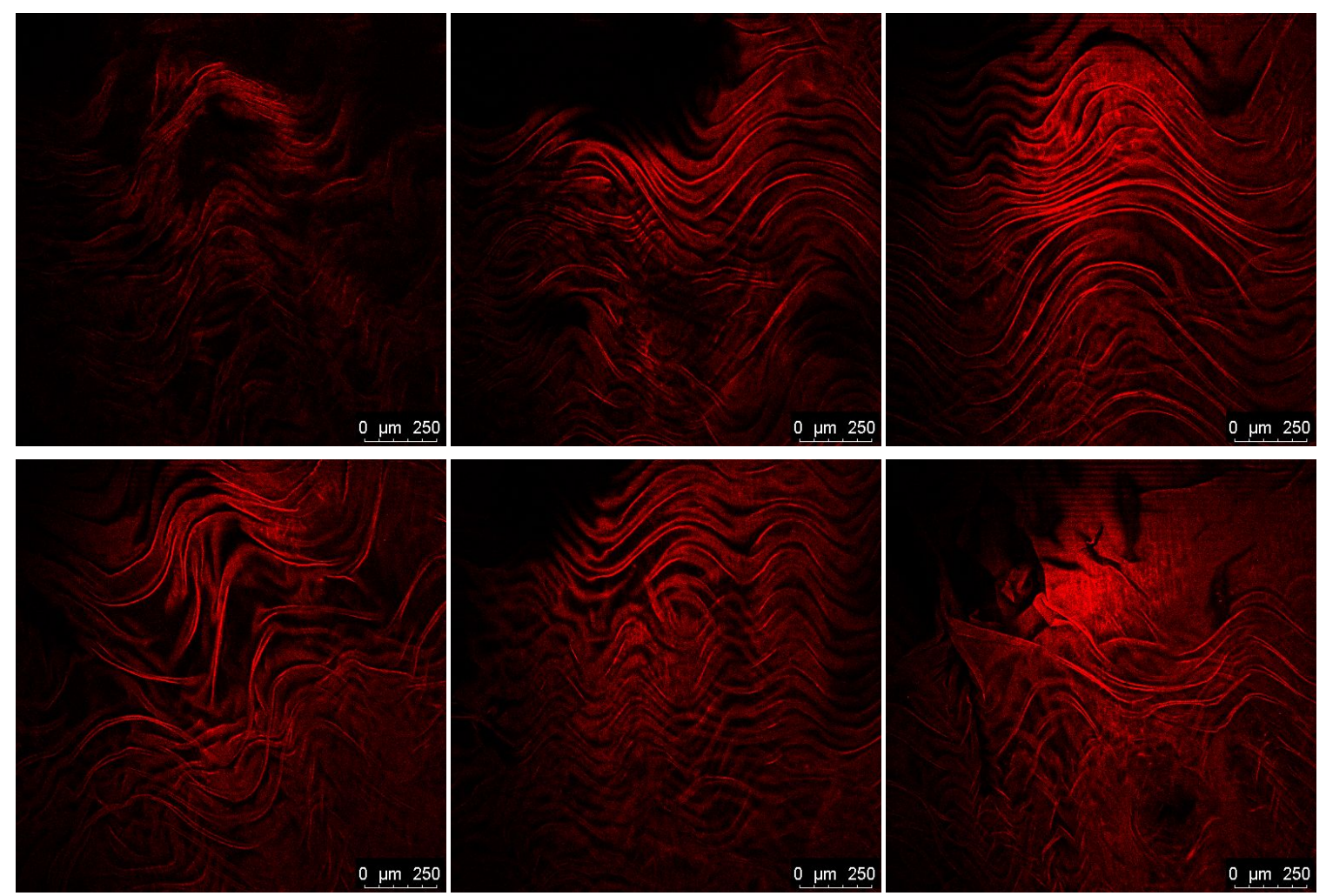

Figure 16. Multiphoton elastin images for unstretched untreated specimens. 
Sample images of elastin extracted from the z-stacks of nicotine-treated specimens are shown in Figure 17.

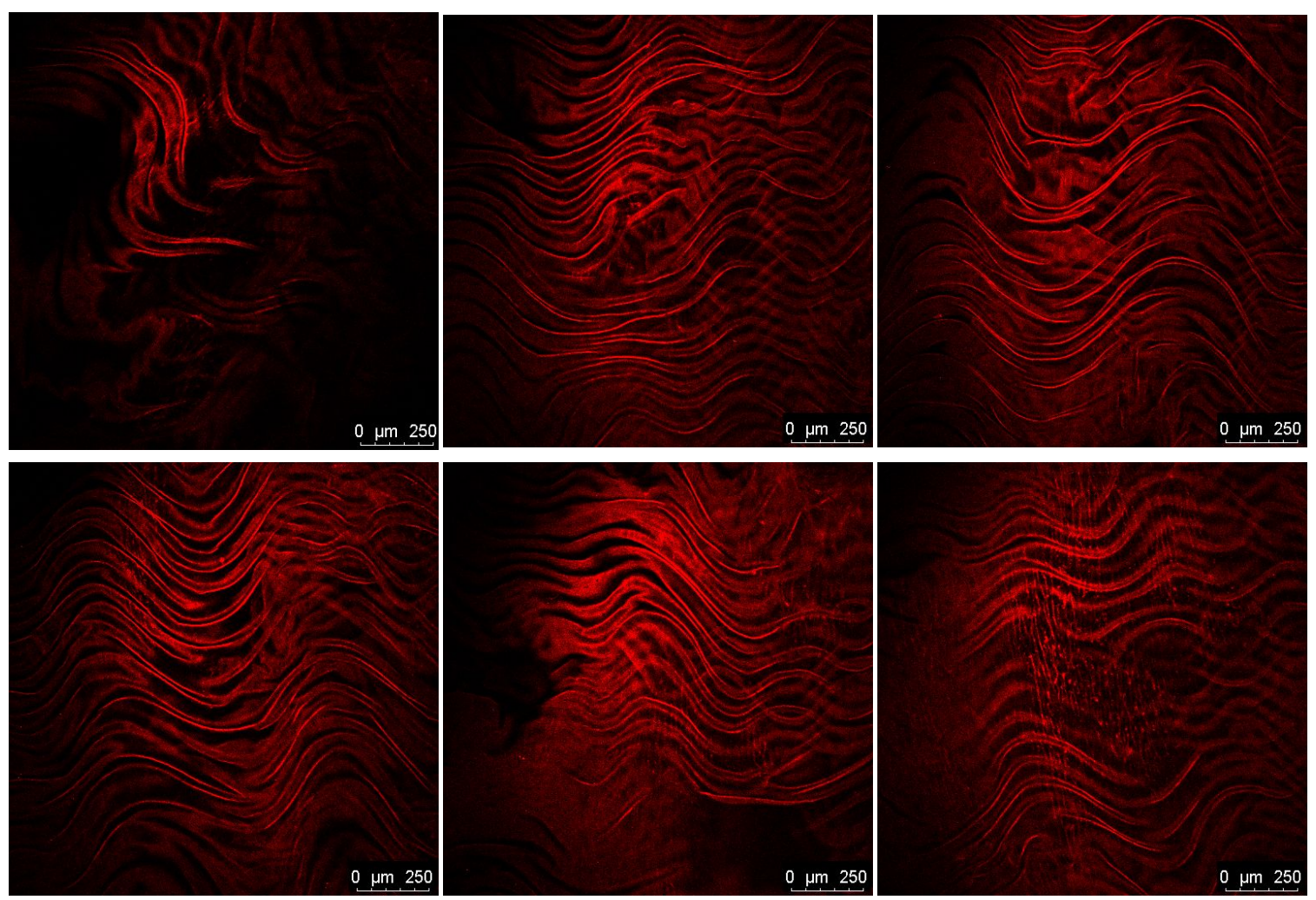

Figure 17. Multiphoton elastin images for unstretched nicotine-treated specimens.

Binary images and skeletonized images were generated for each multiphoton image at the $50 \%$ depth of the associated z-stack. Figure 18 shows the multiphoton input image, binarized image, and skeletonized image for Unstretched Untreated Specimen 3, from one location of the artery. Figure 19 shows the multiphoton input image, binarized image, and skeletonized image for Unstretched Nicotine-Treated Specimen 5, from one location of the artery. 

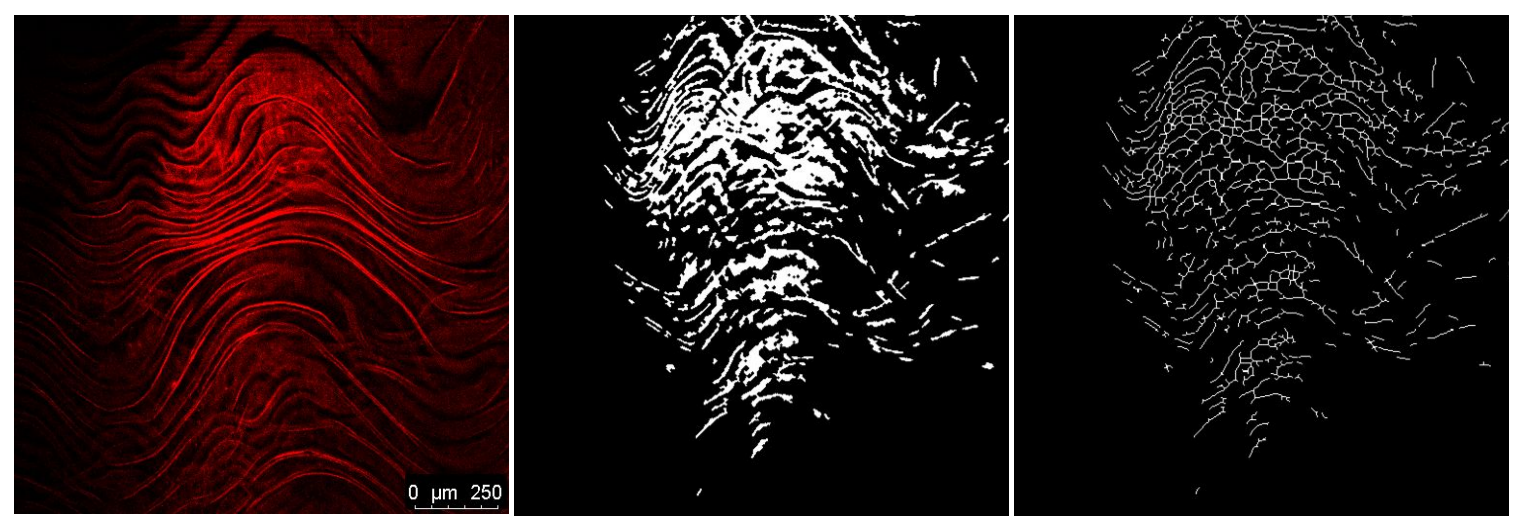

Figure 18. Multiphoton elastin image (left), binarized image (middle), and skeletonized image (right) for Unstretched Untreated Specimen 3.
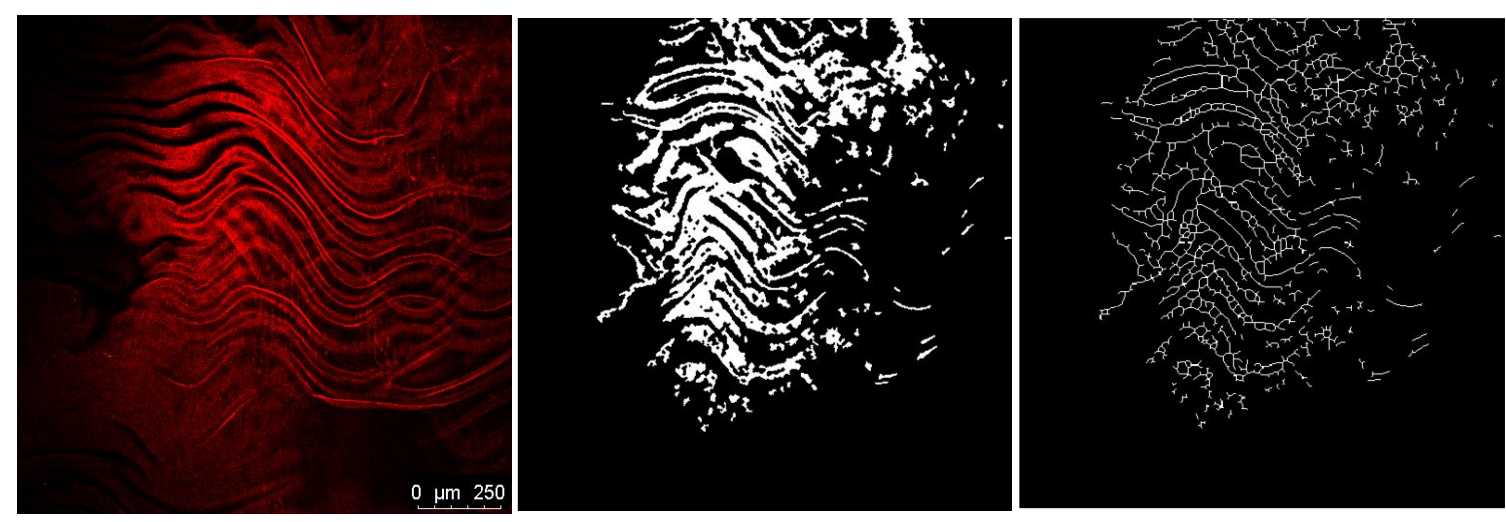

Figure 19. Multiphoton elastin image (left), binarized image (middle), and skeletonized image (right) for Unstretched Nicotine-Treated Specimen 5.

Morphometric parameters extracted from the skeletonized image were mean diameter mean, standard deviation of diameter, characteristic length, standard deviation of characteristic length, and intersection density, using the DiameterJ plug-in of Fiji. The morphometric parameters of unstretched untreated and unstretched nicotine-treated specimens were plotted, shown in Figure 20. 

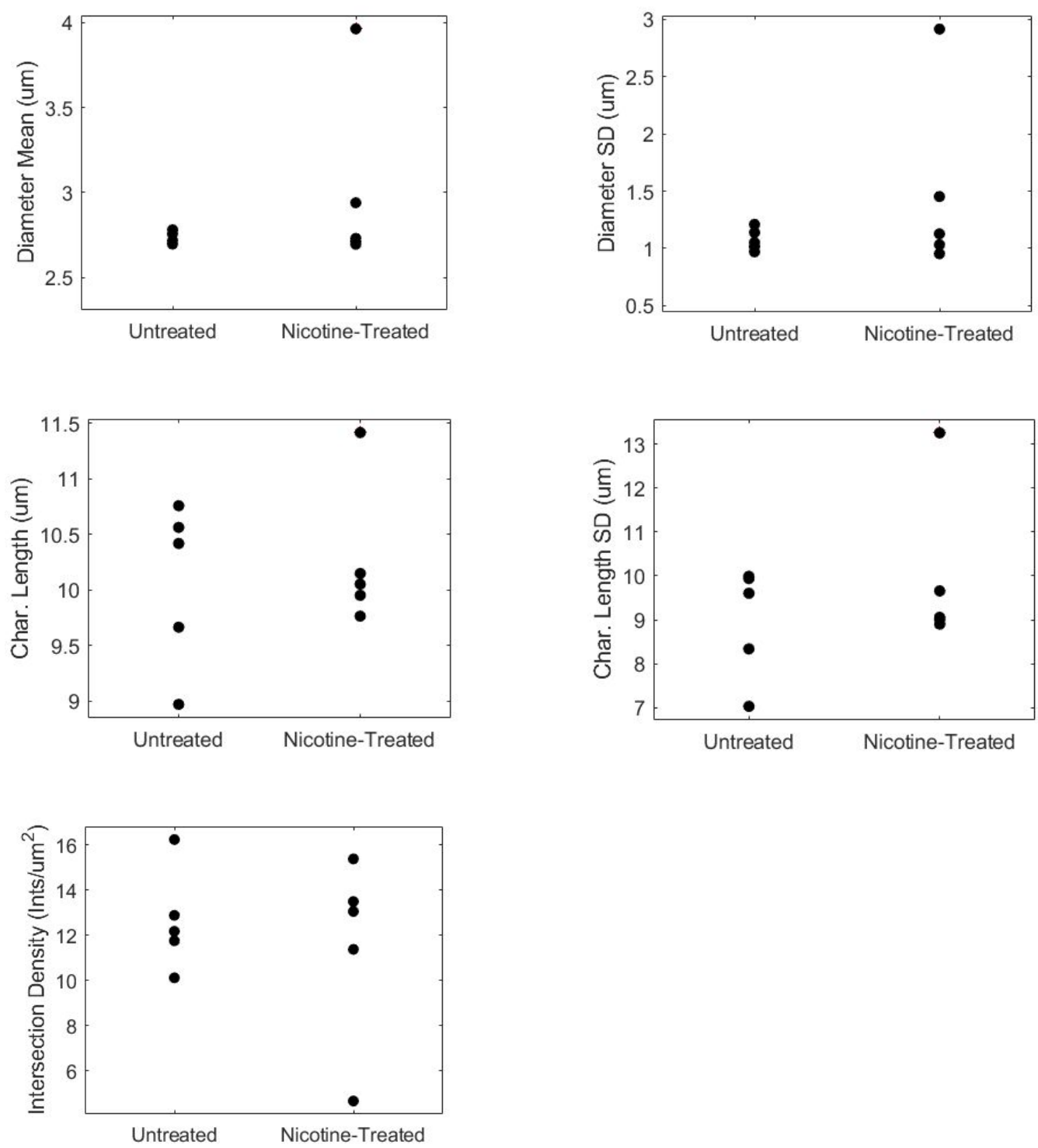

Figure 20. Scatter plots of morphometric parameters extracted from multiphoton images for unstretched untreated and unstretched nicotine-treated specimens. 


\subsection{Experimental Uncertainty}

Sources of experimental uncertainty are as summarized in Table 6 . The nominal value for force was based on the average maximum force of all specimens during actuation. The nominal values for size dimensions were based on average measurements for all ten specimens.

Table 6. Sources of Experimental Uncertainty.

\begin{tabular}{|l|c|c|l|}
\hline Source & Nominal Value & Uncertainty & Notes \\
\hline Force & $0.127 \mathrm{~N}$ & $\pm 0.005 \mathrm{~N}$ & $\begin{array}{l}\text { Average maximum force recorded } \\
\text { during actuation }\end{array}$ \\
\hline Specimen thickness & $40.8 \mu \mathrm{m}$ & $\pm 5 \mu \mathrm{m}$ & Average thickness of specimens \\
\hline Specimen length & $9.24 \mathrm{~mm}$ & $\pm 0.5 \mathrm{~mm}$ & $\begin{array}{l}\text { Average length based on size of } \\
\text { luminum clamps }\end{array}$ \\
\hline Cycle time & $1.8 \mathrm{~s}$ & $\pm 0.2 \mathrm{~s}$ & Per cycle on normal run \\
\hline Strain & 1.0 & \pm 0.2 & Strain recorded during stress relaxation \\
\hline
\end{tabular}

Using the nominal values of max force, thickness and length, the nominal value of maximum stress was $336.9 \mathrm{kPa}$. Using Equation 12, the uncertainty of the specimen thickness, the specimen length and maximum force recorded during actuation, the amount of uncertainty for maximum stress applied to the specimen was $\pm 47.04 \mathrm{kPa}$.

Based on photographs captured during stress relaxation, uncertainty of strain can occur as much as $20 \%$ due to deflection of the load cell. Uncertainty measurements were taken from stress relaxation images due to the static hold of the aluminum clamps, which allowed for ease of measurements. 


\section{CONCLUSIONS}

Based on the results of this research, differences in fatigue parameters were apparent between fatigue tested specimens, untreated and nicotine-treated. On average, nicotine-treated specimens exhibited a higher parameter $A$, or peak stress, by $108 \%$; higher parameter $B$, or loss of tension, by $118 \%$; higher parameter $C$, or degradation slope, by $10.4 \%$; and a higher value of parameter $D$, or width of oscillation band, by $107 \%$. The percentage differences of the fatigue parameters indicate that although differences in peak stresses for both untreated and nicotine-treated specimens are apparent, other characteristics of the mechanical behavior can also be quantified through fatigue testing. Static testing distinguishes differences in peak stress, but does not provide information in regards to parameters such as loss of tension, degradation slope, or width of oscillation band, which supports the hypothesis that fatigue testing reveals arterial characteristics not evident in static testing.

Morphological parameter extraction was performed to analyze the multiphoton microscopy images of unstretched untreated and unstretched nicotine-treated specimens. Images of elastin were converted to binary images in MATLAB, and subsequently, skeletonized using the Fiji plug-in DiameterJ to quantify parameters mean diameter, standard deviation of diameter, characteristic length, standard deviation of characteristic length, and intersection density. This was performed in hopes of understanding the overall mapping of the elastin and potential damage the effect of nicotine has on the fibers. 


\section{REFERENCES CITED}

[1] A. N. Lyle and U. Raaz, "Killing Me Unsoftly: Causes and Mechanisms of Arterial Stiffness," Arterioscler. Thromb. Vasc. Biol., vol. 37, no. 2, pp. e1-e11, Feb. 2017.

[2] J. J. Oliver and D. J. Webb, "Noninvasive assessment of arterial stiffness and risk of atherosclerotic events," Arterioscler. Thromb. Vasc. Biol., vol. 23, no. 4, pp. 554-566, Apr. 2003.

[3] D. A. Vorp, "Biomechanics of abdominal aortic aneurysm," J. Biomech., vol. 40, no. 9, pp. 1887-1902, Jan. 2007.

[4] G. A. Holzapfel, T. C. Gasser, and R. W. Ogden, "A New Constitutive Framework for Arterial Wall Mechanics and a Comparative Study of Material Models," Journal of elasticity and the physical science of solids, vol. 61, no. 1, pp. 1-48, Jul. 2000.

[5] M.-J. Chow, R. Turcotte, C. P. Lin, and Y. Zhang, “Arterial extracellular matrix: a mechanobiological study of the contributions and interactions of elastin and collagen,” Biophys. J., vol. 106, no. 12, pp. 2684-2692, Jun. 2014.

[6] A. Zoumi, X. Lu, G. S. Kassab, and B. J. Tromberg, "Imaging coronary artery microstructure using second-harmonic and two-photon fluorescence microscopy," Biophys. J., vol. 87, no. 4, pp. 2778-2786, Oct. 2004.

[7] A. Bhatnagar et al., "New and Emerging Tobacco Products and the Nicotine Endgame: The Role of Robust Regulation and Comprehensive Tobacco Control and Prevention: A Presidential Advisory From the American Heart Association," Circulation, p. CIR0000000000000669, Mar. 2019.

[8] H. Weisbecker, C. Viertler, D. M. Pierce, and G. A. Holzapfel, "The role of elastin and collagen in the softening behavior of the human thoracic aortic media," $J$. Biomech., vol. 46, no. 11, pp. 1859-1865, Jul. 2013.

[9] Y. C. Fung, K. Fronek, and P. Patitucci, "Pseudoelasticity of arteries and the choice of its mathematical expression," Am. J. Physiol., vol. 237, no. 5, pp. H620-31, Nov. 1979.

[10] C. Lally, A. J. Reid, and P. J. Prendergast, "Elastic behavior of porcine coronary artery tissue under uniaxial and equibiaxial tension," Ann. Biomed. Eng., vol. 32, no. 10, pp. 1355-1364, Oct. 2004.

[11] W. Krasny, H. Magoariec, C. Morin, and S. Avril, "Kinematics of collagen fibers in carotid arteries under tension-inflation loading," J. Mech. Behav. Biomed. Mater., vol. 77, pp. 718-726, Jan. 2018.

[12] S. de Gelidi, G. Tozzi, and A. Bucchi, "The Role of Pre-Conditioning Frequency in 
the Experimental Characterization of Hyper-Elastic Materials as Models for Soft Tissue Applications," Int. J. Appl. Mech., vol. 08, no. 05, p. 1650066, Jul. 2016.

[13] D. Remache, M. Caliez, M. Gratton, and S. Dos Santos, "The effects of cyclic tensile and stress-relaxation tests on porcine skin," J. Mech. Behav. Biomed. Mater., vol. 77, pp. 242-249, Jan. 2018.

[14] W. Zhang, H. Y. Chen, and G. S. Kassab, “A rate-insensitive linear viscoelastic model for soft tissues,” Biomaterials, vol. 28, no. 24, pp. 3579-3586, Aug. 2007.

[15] A. B Ramachandra and J. D. Humphrey, "Biomechanical characterization of murine pulmonary arteries," J. Biomech., vol. 84, pp. 18-26, Feb. 2019.

[16] M. R. Labrosse, C. J. Beller, T. Mesana, and J. P. Veinot, "Mechanical behavior of human aortas: Experiments, material constants and 3-D finite element modeling including residual stress," J. Biomech., vol. 42, no. 8, pp. 996-1004, May 2009.

[17] P. B. Dobrin and W. C. Gley, "Elastase, collagenase and the radial elastic properties of arteries," Experientia, vol. 41, no. 8, pp. 1040-1042, Aug. 1985.

[18] L. M. Van Bortel, M. J. Kool, H. A. Boudier, and H. A. Struijker Boudier, "Effects of antihypertensive agents on local arterial distensibility and compliance," Hypertension, vol. 26, no. 3, pp. 531-534, Sep. 1995.

[19] P. B. Dobrin, "Mechanical properties of arteries," Physiol. Rev., vol. 58, no. 2, pp. 397-460, Apr. 1978.

[20] X. Yu, Y. Wang, and Y. Zhang, "Transmural variation in elastin fiber orientation distribution in the arterial wall," J. Mech. Behav. Biomed. Mater., vol. 77, pp. 745-753, Jan. 2018.

[21] W. Krasny, C. Morin, H. Magoariec, and S. Avril, "A comprehensive study of layer-specific morphological changes in the microstructure of carotid arteries under uniaxial load," Acta Biomater., vol. 57, pp. 342-351, Jul. 2017.

[22] W. Wan, J. B. Dixon, and R. L. Gleason Jr, "Constitutive modeling of mouse carotid arteries using experimentally measured microstructural parameters," Biophys. J., vol. 102, no. 12, pp. 2916-2925, Jun. 2012.

[23] Y. Wang, S. Zeinali-Davarani, and Y. Zhang, "Arterial mechanics considering the structural and mechanical contributions of ECM constituents," J. Biomech., vol. 49, no. 12, pp. 2358-2365, Aug. 2016.

[24] Y. Lanir, "A structural theory for the homogeneous biaxial stress-strain relationships in flat collagenous tissues," J. Biomech., vol. 12, no. 6, pp. 423-436, 1979. 
[25] P. N. Watton, Y. Ventikos, and G. A. Holzapfel, "Modelling the mechanical response of elastin for arterial tissue," J. Biomech., vol. 42, no. 9, pp. 1320-1325, Jun. 2009.

[26] M. A. Zulliger, P. Fridez, K. Hayashi, and N. Stergiopulos, "A strain energy function for arteries accounting for wall composition and structure," J. Biomech., vol. 37, no. 7, pp. 989-1000, Jul. 2004.

[27] J. Creechley, "Bi-Directional Fatigue Life Behavior of Bovine Meniscus," Boise State University, 2016.

[28] W. Hwang and K. S. Han, "Fatigue of Composites-Fatigue Modulus Concept and Life Prediction," J. Compos. Mater., vol. 20, no. 2, pp. 154-165, Mar. 1986.

[29] J. B. Washington III, A study of the effects of oxidation and glycation on the cyclic loading response of isolated arterial elastin. University of Maryland, Baltimore County, 2015.

[30] T. Boulesteix et al., "Micrometer scaleEx Vivo multiphoton imaging of unstained arterial wall structure," Cytometry, vol. 69A, no. 1, pp. 20-26, Jan. 2006.

[31] A. M. Stein, D. A. Vader, L. M. Jawerth, D. A. Weitz, and L. M. Sander, “An algorithm for extracting the network geometry of three-dimensional collagen gels," J. Microsc., vol. 232, no. 3, pp. 463-475, Dec. 2008.

[32] J. S. Bredfeldt et al., "Computational segmentation of collagen fibers from second-harmonic generation images of breast cancer," J. Biomed. Opt., vol. 19, no. 1, p. 16007, Jan. 2014.

[33] R. G. Koch et al., "A custom image-based analysis tool for quantifying elastin and collagen micro-architecture in the wall of the human aorta from multi-photon microscopy," J. Biomech., vol. 47, no. 5, pp. 935-943, Mar. 2014.

[34] R. Rezakhaniha et al., "Experimental investigation of collagen waviness and orientation in the arterial adventitia using confocal laser scanning microscopy," Biomech. Model. Mechanobiol., vol. 11, no. 3-4, pp. 461-473, Mar. 2012.

[35] T. Lu, Z. Chen, H. J. Qi, and T. J. Wang, "A micro-structure based constitutive model for anisotropic stress-strain behaviors of artery tissues," Int. J. Solids Struct., Jan. 2018.

[36] E. A. Murphy, D. Danna-Lopes, I. Sarfati, S. K. Rao, and J. R. Cohen, "Nicotine-stimulated elastase activity release by neutrophils in patients with abdominal aortic aneurysms," Ann. Vasc. Surg., vol. 12, no. 1, pp. 41-45, Jan. 1998.

[37] M. J. Kool, A. P. Hoeks, H. A. Struijker Boudier, R. S. Reneman, and L. M. Van 
Bortel, "Short- and long-term effects of smoking on arterial wall properties in habitual smokers," J. Am. Coll. Cardiol., vol. 22, no. 7, pp. 1881-1886, Dec. 1993.

[38] K. M. Mata et al., "Combining two potential causes of metalloproteinase secretion causes abdominal aortic aneurysms in rats: a new experimental model," Int. J. Exp. Pathol., vol. 92, no. 1, pp. 26-39, Feb. 2011.

[39] A. L. B. Jacob-Ferreira et al., "Evidence for the involvement of matrix metalloproteinases in the cardiovascular effects produced by nicotine," Eur. $J$. Pharmacol., vol. 627, no. 1-3, pp. 216-222, Feb. 2010.

[40] M. U. Wagenhäuser et al., "Chronic Nicotine Exposure Induces Murine Aortic Remodeling and Stiffness Segmentation-Implications for Abdominal Aortic Aneurysm Susceptibility." 31-Oct-2018.

[41] R. M. Hackett, "Strain-Energy Functions," in Hyperelasticity Primer, R. M. Hackett, Ed. Cham: Springer International Publishing, 2018, pp. 19-28.

[42] N. Gundiah, M. B. Ratcliffe, and L. A. Pruitt, "The biomechanics of arterial elastin," J. Mech. Behav. Biomed. Mater., vol. 2, no. 3, pp. 288-296, Jul. 2009.

[43] J. E. Shigley, Shigley's mechanical engineering design. Tata McGraw-Hill Education, 2011.

[44] A. Sciacchitano and B. Wieneke, "PIV uncertainty propagation," Meas. Sci. Technol., vol. 27, no. 8, p. 084006, Jun. 2016.

[45] C. Zaragoza et al., "Animal models of cardiovascular diseases," J. Biomed. Biotechnol., vol. 2011, p. 497841, Feb. 2011.

[46] S. de Gelidi, G. Tozzi, and A. Bucchi, "The effect of thickness measurement on numerical arterial models," Mater. Sci. Eng. C Mater. Biol. Appl., vol. 76, pp. 1205-1215, Jul. 2017.

[47] W. Eng, M. Kim, A. Ramasubramanian, and S.-J. J. Lee, "A Modular Test Platform for Micromechanical Tensile Testing of Soft Biomaterials," in ASME 2018 International Mechanical Engineering Congress and Exposition, 2018, pp. V003T04A030-V003T04A030.

[48] American society for testing and materials, ASTM E606-04 Standard Practice for Strain-Controlled Fatigue Testing2001. ASTM, 1992.

[49] H. Tomizawa, M. Yamazaki, K. Kunika, M. Itakura, and K. Yamashita, "Association of elastin glycation and calcium deposit in diabetic rat aorta," Diabetes Res. Clin. Pract., vol. 19, no. 1, pp. 1-8, Jan. 1993. 
[50] J. Schindelin et al., "Fiji: an open-source platform for biological-image analysis," Nat. Methods, vol. 9, no. 7, pp. 676-682, Jun. 2012.

[51] American society for testing and materials, ASTM E739-10 Standard Practice for Statistical Analysis of Linear Or Linearized Stress-Life (S-N) and Strain-Life ([epsilon]-N) Fatigue Data. ASTM, 2010. 


\section{APPENDIX A: ALUMINUM CLAMPS FABRICATION DRAWINGS}
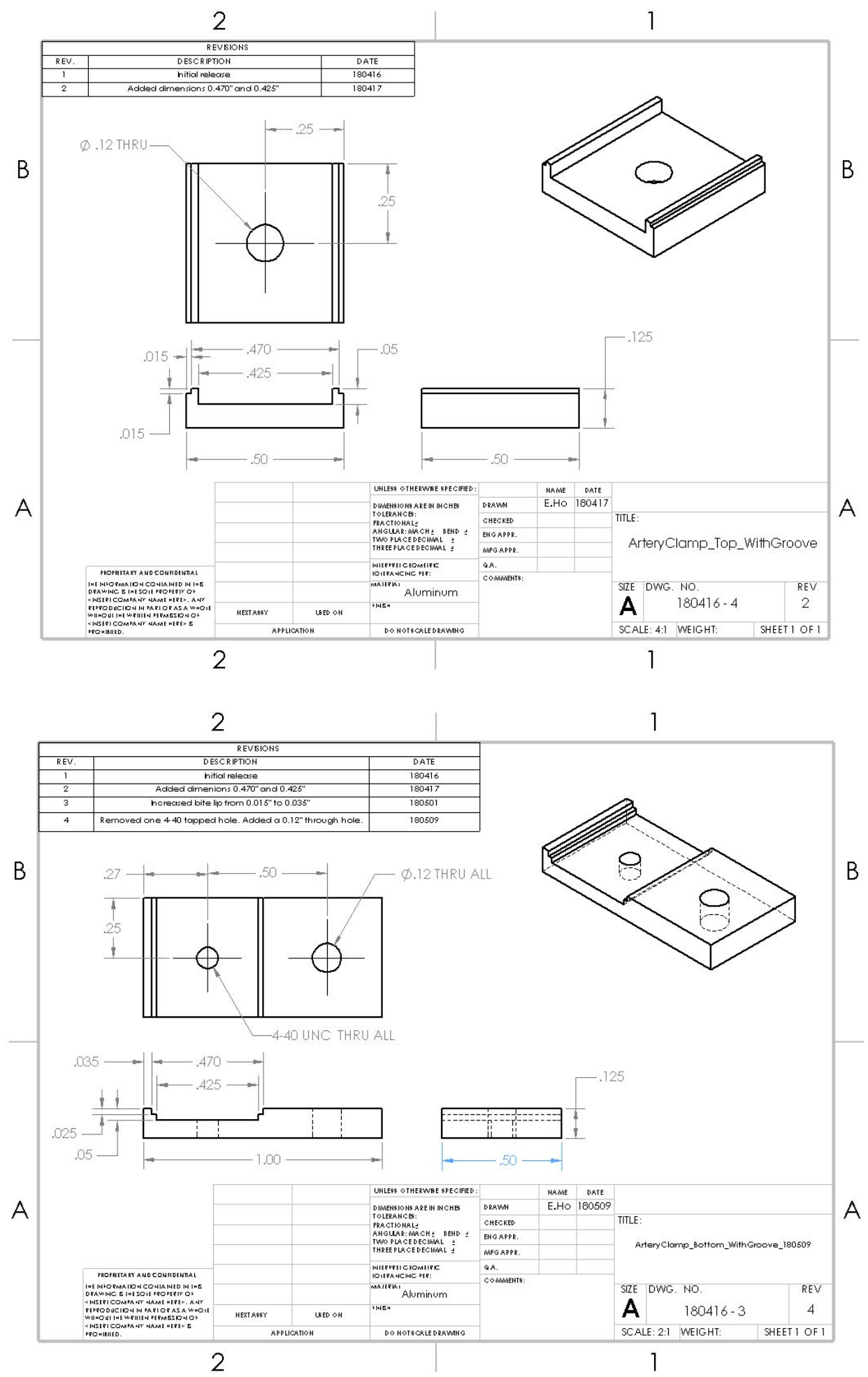


\section{APPENDIX B: RAW DATA OF FIT PARAMETERS}

Fit parameters for untreated specimens, according to model explained in Section 4.4.

\begin{tabular}{|c|c|c|c|c|c|}
\hline \multirow{7}{*}{} & Specimen & $A$ & $B$ & $C$ & $D$ \\
\cline { 2 - 6 } & 1 & 196 & 87.1 & 0.021 & 64.1 \\
\cline { 2 - 6 } & 2 & 284 & 139 & 0.032 & 82.5 \\
\cline { 2 - 6 } & 3 & 269 & 132 & 0.015 & 82.4 \\
\cline { 2 - 6 } & 4 & 232 & 104 & 0.017 & 70.9 \\
\cline { 2 - 6 } & 5 & 253 & 111 & 0.028 & 74.8 \\
\hline
\end{tabular}

Fit parameters for nicotine-treated specimens, according to model explained in Section 4.4.

\begin{tabular}{|c|c|c|c|c|c|}
\hline & Specimen & $A$ & $B$ & $C$ & $D$ \\
\cline { 2 - 6 } & 1 & 596 & 295 & 0.027 & 175 \\
\cline { 2 - 6 } & 2 & 457 & 212 & 0.023 & 145 \\
\cline { 2 - 6 } $\begin{array}{c}\text { Nicotine- } \\
\text { Treated }\end{array}$ & 3 & 341 & 156 & 0.020 & 113 \\
\cline { 2 - 6 } & 4 & 665 & 333 & 0.034 & 192 \\
\cline { 2 - 6 } & 5 & 512 & 255 & 0.021 & 151 \\
\hline
\end{tabular}

